Seguridad Operacional y Logística Aeronáutica

Operational Safety and Aviation Logistics

Segurança Operacional e Logística Aeronáutica

\title{
Evaluación estructural alar de aeronave Categoría VLA PARA CERTIFICACIÓN EN COLOMBIA ${ }^{1}$
}

Wing's StRUCTURAL ASSESSMENT OF A VLA AIRCRAFt FOR CERTIFICATION IN COLOMBIA ${ }^{2}$

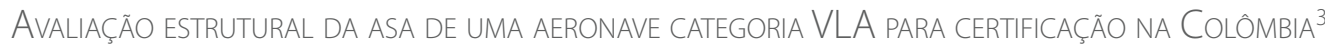

Andrés Palomino ${ }^{4}$

World Aircraft Company South America - WACSA S.A.S. Cali, Colombia

\section{CIENCIA Y PODER AÉREO}

ISSN 1909-7050 / E- ISSN 2389-9468 / Volumen 13 / Número 2 / julio-diciembre de 2018/ Colombia /pp. 28-45 Recibido: 13/08/2018

Aprobado par evaluador: 29/08/2018

Doi: 10.18667/cienciaypoderaereo.600 
Para citar este artículo:

Palomino, A. (2018). Evaluación estructural alar de aeronave categoría VLA para certificación en Colombia. Ciencia y Poder Aéreo, 13(2), 28-45. doi: 10.18667/cienciaypoderaereo.600

\footnotetext{
' Reporte de caso asociado al proyecto de Certificacion Bajo Norma Cs-Vla De Aeronave Colombiana.

${ }^{2}$ Report of case associated to the project of Certification Under Norm Cs-Vla of Colombian Aircraft.

${ }^{3}$ Relato de caso associado ao Projeto de Certificação sob o Padrão de Aeronaves Colombianas Cs-VIa.

${ }^{4}$ Ingeniero aeronáutico e ingeniero mecánico egresado de la Universidad Pontificia Bolivariana de Medellín (UPB). Actualmente trabaja en la obtención de certificado de aeronavegabilidad especial para la aeronave colombiana WA500-AG de la empresa World Aircraft Company, South America (WACSA) S. A. S. Cali, Colombia. ORCID: 0000-0002-0518-3833. Correo electrónico: andrepalcar@hotmail.com
}

Resumen: El presente reporte de caso muestra el trabajo y los resultados de la evaluación estructural del ala llevado a cabo sobre la aeronave de diseño y fabricación colombiana WA500-AG. El proceso consistió en la apropiación de una norma de diseño, el cálculo de las cargas estructurales, la identificación de los casos de carga más críticos, la construcción de un banco y un espécimen de pruebas, el sometimiento de la estructura a las cargas calculadas y la identificación y corrección de aspectos de diseño. Como resultado, se obtiene un ala que demuestra ser capaz de soportar los requerimientos estructurales, dando así cumplimiento a la normativa vigente. Todo este trabajo, está enmarcado en lo que sería la primera certificación de una aeronave nacional categoría VLA ante la Unidad Administrativa Especial de la Aeronáutica Civil.

Palabras clave: aeronave VLA, análisis estructural, certificación aeronáutica, pruebas estructurales de ala.

Abstract: This case report shows the work and results of wing's structural assessment carried out on the Colombian design and manufacturing aircraft WA500-AG. The process consisted of the appropriation of a design standard, the calculation of structural loads, the identification of the most critical load cases, the construction of a bench and a test specimen, subjecting the structure to the calculated loads and the identification and adjustments of design aspects. As a result, it is obtained a wing able to support the structural requirements thus complying with current regulations. All this work is framed in what would be the first certification of a national aircraft category VLA before the Colombian Civil Aviation Authority.

Keywords: VLA aircraft, structural analysis, aircraft certification, wing structural tests.

Resumo: O presente artigo apresenta os trabalhos e resultados da avaliação estrutural efetuada sobre a asa da aeronave WA500-AG, um aparelho de concepção e fabricação colombiana. O processo consistiu na apropriação de uma norma de projeto, o computo das cargas estruturais, a identificação dos casos de carga mais críticos, a construção de um banco e amostra de ensaio, o teste da estrutura ás cargas calculadas e a identificação e correção de aspectos de design. Como resultado, uma assa que prova suportar os requerimentos estruturais é obtida, dando assim cumprimento à regulamentação vigente. Tudo o referido trabalho, enquadrado em o que seria a primeira certificação de uma aeronave categoria VLA feita pela Agência de Aviação Civil da Colômbia.

Palavras-chave: aeronave VLA, analise estrutural, certificação aeronáutica, ensaios estruturais de asas. 


\section{Introducción}

La fabricación, comercialización y operación de aeronaves ligeras se hacía con relativa facilidad, hasta hace un poco más de una década, ya que no existían normas internacionales que regularan esta categoría de aeronaves. Las regulaciones de la $\mathrm{OACl}$ en relación a los requerimientos de aeronavegabilidad, establece en la parte $V$ del anexo 8 los requisitos de diseño que deben cumplir aeronaves "pequeñas", definiendo estas como aquellas con pesos máximo de despegue entre $750 \mathrm{~kg}$ y 5700 kg, permitiendo y responsabilizando entonces a cada Estado de definir los requisitos de aeronavegabilidad para aeronaves con pesos inferiores (OACl, 2010).

Ante la creciente demanda de aeronaves ligeras para recreación privada y actividades especiales como aspersión agrícola, la Administración Federal de Aviación Americana (FAA), así como la Agencia Europea de Seguridad de Aviación (EASA), hacen efectivas entre 2003 y 2004 nuevas regulaciones que acogen al tipo de aeronaves denominadas Light Sport Aircraft (LSA) o Very Light Aircraft (VLA).

Por otra parte en Colombia, la Aeronáutica Civil establece que mientras se adopta un código de aeronavegabilidad propio de aeronaves ligeras, para efectos de certificación, los fabricantes colombianos que se acojan al estándar de aeronavegabilidad europeo EASA Certification Specifications for Very Light Aeroplanes (CS-VLA) podrán obtener un certificado de aeronavegabilidad especial respecto a dicho diseño (Aerocivil, 2012).

En dicha resolución se definen las características que debe cumplir una aeronave para ser considerada de categoría VLA:

- Poseer un peso máximo de despegue no superior a $750 \mathrm{~kg}$, sin importar el tipo de estructura (tubular, monocoque, etc.), alas en tela, lámina o material compuesto.

- Velocidad de pérdida no superior a $83.34 \mathrm{~km} / \mathrm{h}$ (45 kts) en configuración de aterrizaje.

- Un solo motor certificado, ya sea del tipo recíproco, turbina, a reacción o eléctrico.

- Una capacidad máxima de 2 asientos, incluyendo el piloto.

- Una hélice de paso fijo o variable.

- Cabina cerrada no presurizada.

- Certificado bajo reglas de vuelo visual diurno (VFR).
World Aircraft Company South America es una empresa colombiana derivada de Agrocopteros Ltda., que cuenta con más de 30 años de experiencia diseñando, fabricando y comercializando aeronaves cuyas características las encuadran en la categoría VLA.

Por lo tanto, el presente reporte de caso exhibe parte del proceso de certificación, consistente en la aplicación de los requerimientos estructurales de la norma CS-VLA, el cálculo de las cargas en el ala, la identificación de los casos de carga más críticos, la construcción de un banco y espécimen de pruebas, el sometimiento de la estructura a las fuerzas calculadas, los ajustes de diseño y la presentación de la evidencia de cumplimiento ante la Aeronáutica Civil Colombiana. Todo este proceso hecho sobre la aeronave WA500-AG, un aparato concebido por el ingeniero colombiano Maximo Tedesco y que actualmente se encuentra en la fase de pruebas de vuelo (figura 1 y 2).

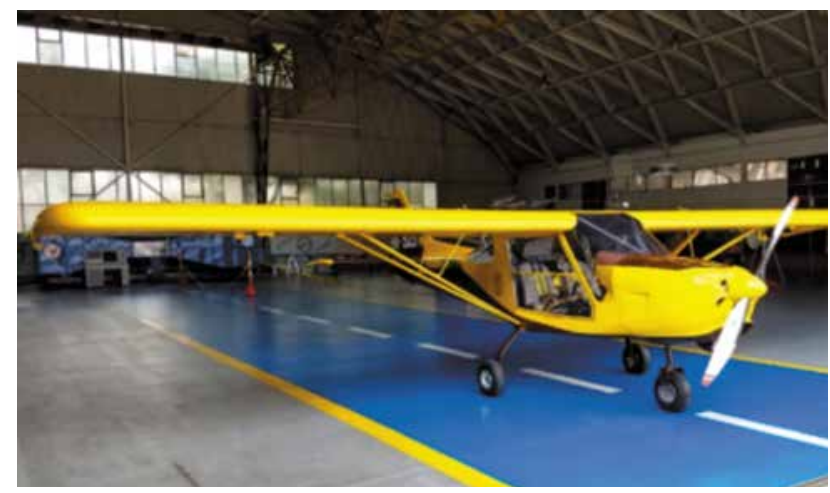

Figura 1. WA500-AG en los hangares de la Escuela Militar de Aviación (EMAVI) de la Base Aérea Marco Fidel Suarez de Cali, Colombia

Fuente: WACSA S. A. S (2015).

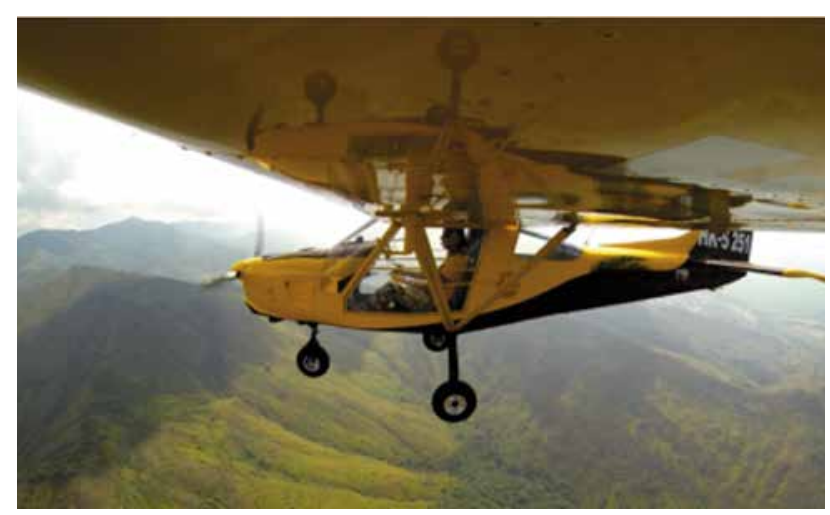

Figura 2. WA500-AG en vuelo Fuente: WACSA S. A. S (2015). 
En la tabla 1 se presentan algunas especificaciones de diseño de la aeronave WA500-A

Tabla 1.

Especificaciones de diseño

\begin{tabular}{ll}
\hline Envergadura & $10[\mathrm{~m}]$ \\
\hline Cuerda Alar & $1.25[\mathrm{~m}]$ \\
\hline Área Alar & $12.5\left[\mathrm{~m}^{2}\right]$ \\
\hline Longitud & $6.5[\mathrm{~m}]$ \\
\hline Altura & $2.45[\mathrm{~m}]$ \\
\hline Peso vacío & $330[\mathrm{~kg}]$ \\
\hline Peso bruto máximo de operación & $690[\mathrm{~kg}]$ \\
\hline Motor & Rotax 912 S2 de 100 [hp] \\
& (Certificado) \\
\hline Hélice & MT Propeller bipala 68" \\
& (Certificada) \\
\hline
\end{tabular}

Fuente: elaboración propia.

Con relación al desempeño, en la tabla 2 se exponen algunas características importantes.

Tabla 2.

Características de desempeño

\begin{tabular}{ll}
\hline Distancia para el despegue & $70[\mathrm{~m}]$ \\
\hline Distancia para aterrizaje & $100[\mathrm{~m}]$ \\
\hline Velocidad crucero al $75 \%$ & $105[\mathrm{mph}]$ \\
\hline Velocidad máxima & $125[\mathrm{mph}]$ \\
\hline Velocidad de pérdida con flaps & $30[\mathrm{mph}]$ \\
\hline Velocidad de pérdida sin flaps & $35[\mathrm{mph}]$ \\
\hline Techo de servicio & $14000[\mathrm{ft}](4270[\mathrm{~m}])$ \\
\hline Alcance & $460[\mathrm{mi}]$ \\
\hline Autonomía & $4.4[\mathrm{~h}]$ \\
\hline
\end{tabular}

Fuente: elaboración propia.

\section{Estimación de cargas}

Las cargas tomadas en consideración para el presente proyecto son básicamente dos: cargas aerodinámicas y cargas inerciales. Las primeras están relacionadas a las velocidades y características geométricas propias de la aeronave, por lo que para el cálculo de estas, se parte de resultados aerodinámicos previos (WACSA S. A. S, 2015), mientras que las inerciales son aquellas debido a la masa y el cómputo de estas, simplemente se hace multiplicando el peso por factores de carga o fuerzas $G$ dados por norma.
La normativa relacionada a los requisitos estructurales está definida dentro del CS-VLA Subpart C - Structures (EASA, 2009). Por lo tanto, esta es la parte de la norma en la que se enfoca el presente reporte.

\section{Envolvente de vuelo}

Ambos diagramas $V$ - $n$, el de maniobra y de ráfagas son construidos de acuerdo a los lineamientos dados en los numerales CS-VLA 333 al CS-VLA 341.

Las principales velocidades de diseño se calculan de acuerdo a la definición dada en CS-VLA 335:

$$
\begin{gathered}
V_{c}=2.4 * \sqrt{\frac{M * g}{S}}[\mathrm{~m} / \mathrm{s}] \\
V_{d}=1.25 * V_{c} \\
V_{a}=V_{s} * \sqrt{n}
\end{gathered}
$$

Siendo:

$$
\begin{aligned}
M & =\text { Masa de la aeronave }[\mathrm{kg}] \\
g & =\text { Aceleración de la gravedad }\left[\mathrm{m} / \mathrm{s}^{2}\right] \\
\mathrm{S} & =\text { Área alar }[\mathrm{m} 2] \\
V_{s} & =\text { Velocidad de pérdida }[\mathrm{m} / \mathrm{s}] \\
n & =\text { Factor de carga }
\end{aligned}
$$

Los resultados obtenidos son:

$$
\begin{aligned}
& V c=46.08[\mathrm{~m} / \mathrm{s}] \\
& V d=57.60[\mathrm{~m} / \mathrm{s}] \\
& V a=42.88[\mathrm{~m} / \mathrm{s}]
\end{aligned}
$$

Por normativa, los factores de carga límites están definidos en CS-VLA 337 como:

$$
\begin{aligned}
& \mathrm{n}(+)=3.8 \\
& \mathrm{n}(-)=-1.5
\end{aligned}
$$

Y la curva de pérdida de la envolvente de vuelo se construye con ayuda de la siguiente expresión:

$$
n=\frac{\frac{1}{2} * \rho * S * C L_{M A X} * V^{2}}{M * g}
$$


El diagrama V-n de maniobras resultante está representado en la figura 3.

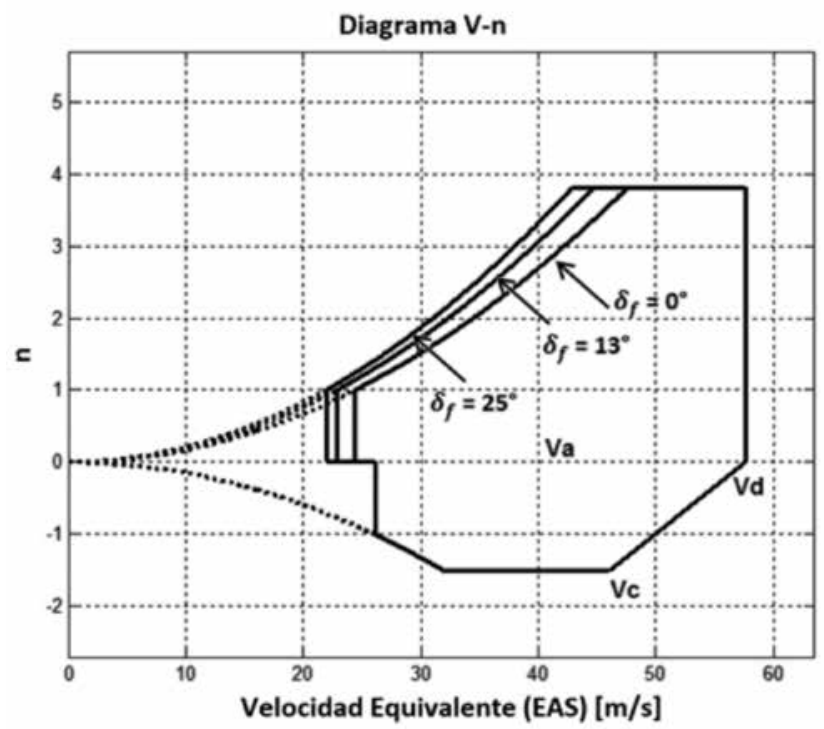

Figura 3. Diagrama V-n de maniobras para peso máximo y diferentes posiciones de flap

Fuente: elaboración propia por medio de las herramientas Matlab ${ }^{\circledast}$ y Excel ${ }^{\circledR}$.

Por su parte, el factor de carga debido a ráfagas está definido en CS-VLA 341 como:

$$
n=1+\frac{\frac{1}{2} * \rho_{o} * V * a * K_{g} * U_{d e}}{M * g / S}
$$

Siendo:

$$
\begin{aligned}
& K_{g} \quad= \\
& \frac{0.88 * \mu_{g}}{5.3+\mu_{g}} \\
& \mu_{g}=\frac{2 *\left(\frac{M}{S}\right)}{\rho * \bar{c} * a} \\
& \mathrm{M}=\text { Masa de la aeronave }[\mathrm{kg}] \\
& \rho_{0}=\text { Densidad del aire al nivel del mar }[\mathrm{kg} / \mathrm{m} 3] \\
& \rho=\text { Densidad del aire }[\mathrm{kg} / \mathrm{m} 3] \\
& c=\text { Cuerda media aerodinámica }[\mathrm{m}] \\
& \mathrm{S}=\text { Área alar }\left[\mathrm{m}^{2}\right] \\
& g=\text { Aceleración de la gravedad }\left[\mathrm{m} / \mathrm{s}^{2}\right] \\
& V \quad=\quad \text { Velocidad }[\mathrm{m} / \mathrm{s}] \\
& a \quad=\quad \text { Pendiente de la curva de sustentación }\left[\operatorname{Rad}^{-1}\right] \\
& U_{\text {de }} \quad=\quad \text { Velocidades de ráfaga derivadas, definidas en } \\
& \text { CS-VLA } 333 \text { (1)(i) y (1)(ii) }
\end{aligned}
$$

Los factores de carga de ráfagas son calculados tanto para una condición de peso máximo como de peso mínimo (ver tabla 3):

Tabla 3.

Resultados para el diagrama $\vee$-n de ráfagas

\begin{tabular}{ccccc}
\hline$M[k g]$ & 432 & 432 & 693 & 693 \\
\hline$V[\mathrm{~m} / \mathrm{s}]$ & 46.08 & 57.60 & 46.08 & 57.60 \\
\hline$\mu_{q}$ & 14.11 & 14.11 & 22.64 & 22.64 \\
\hline$K_{q}$ & 0.639 & 0.639 & 0.713 & 0.713 \\
\hline$U_{d e}$ & 15.24 & 7.62 & 15.24 & 7.62 \\
\hline $\mathrm{n}(+)$ & 4.1 & 2.94 & 3.74 & 2.71 \\
\hline $\mathrm{n}(-)$ & -2.11 & -0.94 & -1.73 & -0.71 \\
\hline
\end{tabular}

Fuente: elaboración propia.

Por lo tanto el diagrama $\vee-n$ de ráfagas construido es de la forma que se presenta en la figura 4.:

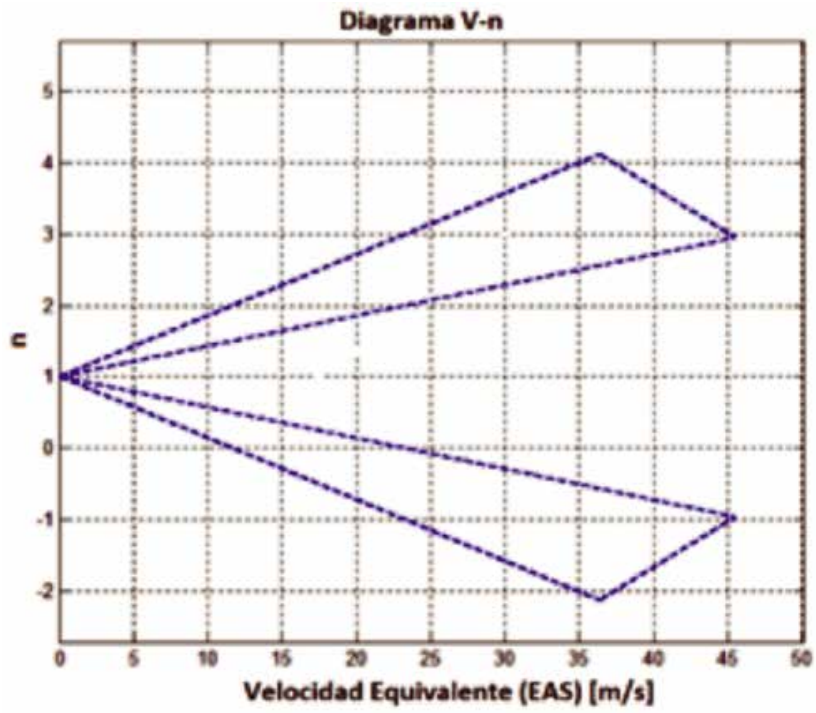

Figura 4. Diagrama V-n de ráfagas

Fuente: elaboración propia por medio de las herramientas Matlab ${ }^{\circledast}$ y Excel ${ }^{\circledast}$.

La combinación de los diagramas de maniobras y de ráfagas definen la envolvente de vuelo, como se puede apreciar en la figura 5: 


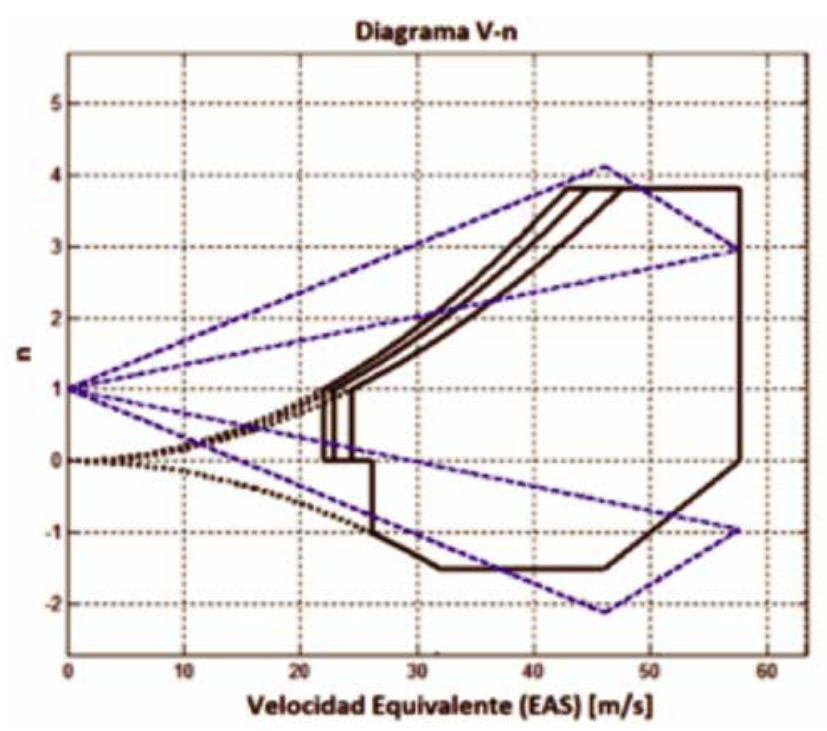

Figura 5. Envolvente de vuelo combinada

Fuente: elaboración propia por medio de las herramientas Mat$1 \mathrm{ab} \mathrm{b}^{\circledast}$ y Excel $^{\circledast}$

Con la información de la envolvente de vuelo final se identifican los puntos de condición más críticos, de acuerdo con CS-VLA 333 (d), como se aprecia en la figura 6:

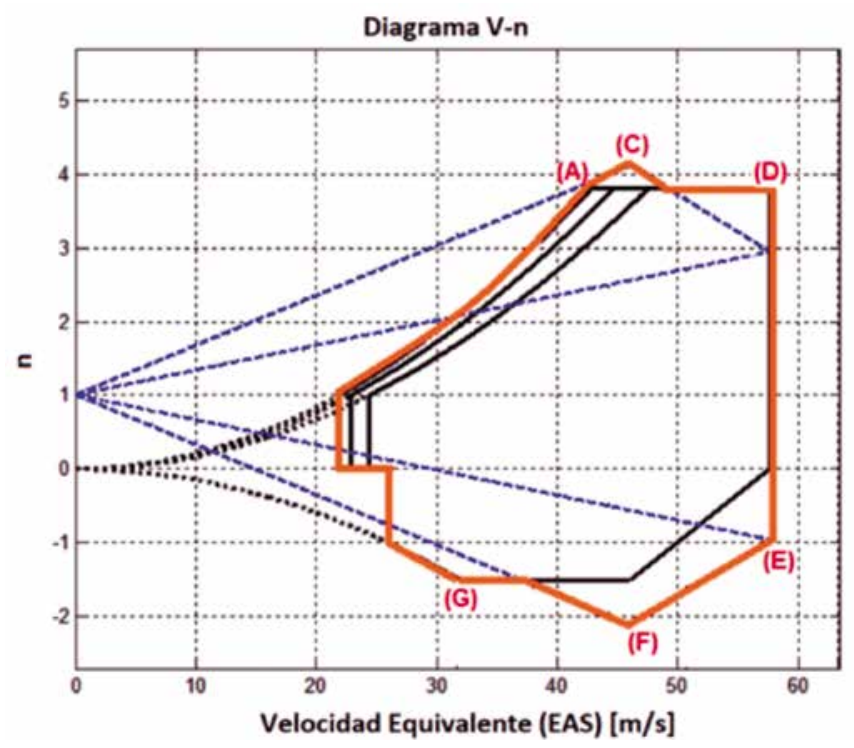

Figura 6. Puntos críticos del diagrama V-n

Fuente: elaboración propia por medio de las herramientas Matlab $^{\oplus}$ y Excel $^{\oplus}$

Por lo tanto, en la tabla 4 se registran las condiciones que se tienen en consideración para el cálculo de cargas como para las pruebas estructurales.
Tabla 4.

Características de los puntos críticos del diagrama V-n

\begin{tabular}{cccc}
\hline $\begin{array}{c}\text { Condición de } \\
\text { vuelo }\end{array}$ & $\begin{array}{c}\text { Factor de } \\
\text { carga }\end{array}$ & $\begin{array}{c}\text { Velocidad } \\
\text { [m/s] }\end{array}$ & Peso [kg] \\
\hline (A) & 3.8 & 42.88 & 693 \\
\hline$(\mathrm{C})$ & 4.1 & 46.08 & 432 \\
\hline$(\mathrm{D})$ & 3.8 & 57.60 & 693 \\
\hline$(\mathrm{E})$ & -0.94 & 57.60 & 432 \\
\hline$(\mathrm{F})$ & -2.11 & 46.08 & 432 \\
\hline$(\mathrm{G})$ & -1.5 & 32.04 & 693 \\
\hline
\end{tabular}

Fuente: elaboración propia.

\section{Determinación de cargas en vuelo}

Las cargas en vuelo actuantes en el ala y empenaje son estimadas balanceando las fuerzas aerodinámicas con las fuerzas inerciales, como se observa en la figura 7.

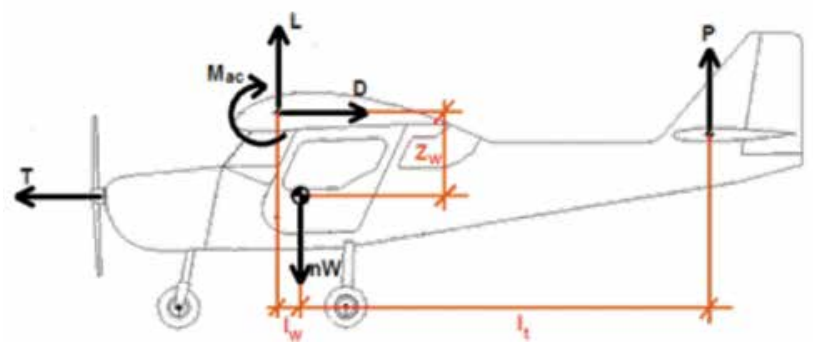

Figura 7. Diagrama de cuerpo libre WA500-AG Fuente: elaboración propia.

Donde:

$$
\begin{array}{lll}
L & = & \text { Sustentación del ala } \\
D & = & \text { Arrastre del ala } \\
P & = & \text { Carga aerodinámica empenaje } \\
T=0 & = & \text { Empuje (igual a } 0 \text { de acuerdo con CS-VLA } \\
& & 345(\mathrm{~d})) \\
W=M * g & = & \text { Peso } \\
M_{a c} & = & \text { Momento respecto al centro aerodinámico } \\
I_{w} & = & \text { (CA) } \\
Z_{w} & = & \text { Distancia horizontal del CA del ala al CG } \\
I_{t} & = & \text { Distancia horizontal del CA de la cola al CG }
\end{array}
$$

Como se observa, el equilibrio vertical implica que:

$$
L+P-n * W=0
$$

Y el equilibrio de momentos respecto al CG de la aeronave:

$$
M_{a c}+L * I_{w}+D * Z_{w}-P * \mid t=0
$$


Para un dado peso, factor de carga y velocidad, las ecuaciones (6) y (7) se pueden resolver para calcular los valores desconocidos de Ly P. Sin embargo, otros parámetros como el arrastre $D$ dependen del coeficiente de sustentación $C_{L^{\prime}}$ que a su vez es función de $L_{;}$por lo que en la práctica, se aplica un método de sucesivas aproximaciones (Megson, 2007), en donde se supone inicialmente que la carga en el empenaje es despreciable $(P=0)$ y se itera hasta que los valores no cambien más allá de un error establecido.

Es importante resaltar que los valores de $L$ y $P$ obtenidos por el anterior método son transformados a fuerzas normales y transversales al ala, de acuerdo con el ángulo de ataque de la condición de vuelo estudiada (figura 8).

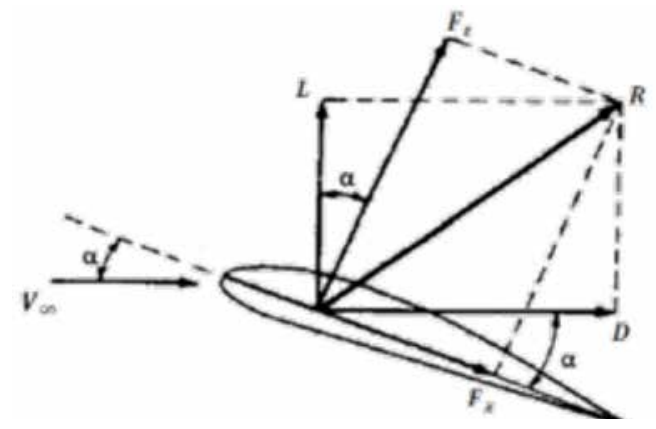

Figura 8. Descomposición de L y P en fuerza normal y transversal Fuente: elaboración propia por medio de las herramientas Mat$\mathrm{lab}^{\circledast}$ y Excel $^{\circledR}$

De la figura 8 se deduce que las ecuaciones de descomposición están dadas por:

$$
\begin{aligned}
& F_{z}=L * \cos (a)+D * \sin (a) \\
& F_{x}=D * \cos (a)-L * \sin (a)
\end{aligned}
$$

Los datos necesarios para computar las cargas son clasificados en 3 tipos: datos dependientes del peso (tabla 5), datos dependientes de la posición del flap (tabla 6) y datos independientes (tabla 7).
Tabla 5.

Datos dependientes del peso

\begin{tabular}{ccc}
\hline \multicolumn{3}{c}{ Datos dependientes del peso } \\
\hline & $\mathbf{G W}=\mathbf{4 3 2}[\mathbf{k g}]$ & $\mathbf{G W}=\mathbf{6 9 3}[\mathbf{k g}]$ \\
\hline $\boldsymbol{I}_{\mathbf{w}}[\mathrm{m}]$ & 0.0648 & 0.1748 \\
\hline $\boldsymbol{I}_{\mathbf{w}}[\mathrm{m}]$ & 3.476 & 3.366 \\
\hline
\end{tabular}

Fuente: elaboración propia.

Tabla 6.

Datos dependientes de la posición del flap

\begin{tabular}{|c|c|c|}
\hline & $\delta_{f}=0^{0}$ & $\delta_{f}=25^{0}$ \\
\hline$C M_{a c}$ & -0.1022 & -0.241 \\
\hline $\begin{array}{l}\text { Ecuación } \\
\text { polar de } \\
\text { arrastre }\end{array}$ & $\begin{array}{c}C_{D}=0.0076363+ \\
0.044226^{*} C_{L}^{2}\end{array}$ & $\begin{array}{c}C_{D}=0.0247153+ \\
0.044226 * C_{L}^{2}\end{array}$ \\
\hline $\begin{array}{l}\text { Ecuación } \\
\text { de la } \\
\text { pendiente de } \\
\text { sustentación } \\
\text { (a en [deg]) }\end{array}$ & $\begin{array}{c}C_{L}=0.08813^{*} a+ \\
0.45827\end{array}$ & $\begin{aligned} C_{L}= & 0.08813^{*} a+ \\
& 1.1545\end{aligned}$ \\
\hline
\end{tabular}

Datos dependientes de la posición del flap

Fuente: elaboración propia.

Tabla 7.

Datos independientes

\begin{tabular}{cc}
\hline \multicolumn{2}{c}{ Datos independientes } \\
\hline$Z_{w}[\mathrm{~m}]$ & 0.590 \\
\hline $\bar{C}[\mathrm{~m}]$ & 1.261 \\
\hline$S\left[\mathrm{~m}^{2}\right]$ & 12.01 \\
\hline$\left[\mathrm{kg} / \mathrm{m}^{3}\right]$ & 1.225 \\
\hline
\end{tabular}

Fuente: elaboración propia.

Un programa que implementa el método de sucesivas aproximaciones se escribe en Matlab ${ }^{\oplus}$, los resultados para cada combinación de peso, factor de carga, velocidad y posición del flap del diagrama $\vee-n$ son presentados en la tabla 8: 
Tabla 8.

Resultados determinación de cargas en vuelo

\begin{tabular}{|c|c|c|c|c|c|c|c|c|}
\hline CONDICIÓN DE VUELO & & & (A) & (C) & (D) & (E) & $(\mathrm{F})$ & (G) \\
\hline Peso & w & {$[\mathrm{kg}]$} & 693 & 432 & 693 & 432 & 432 & 693 \\
\hline Posición del CG & & [\%MAC] & 0.34 & 0.30 & 0.34 & 0.30 & 0.34 & 0.34 \\
\hline \multirow{2}{*}{ Velocidad } & \multirow{2}{*}{ v } & {$[\mathrm{m} / \mathrm{s}]$} & 42.88 & 46.08 & 57.60 & 57.60 & 46.08 & 32.04 \\
\hline & & [mph] & 95.9 & 103.1 & 128.8 & 128.8 & 103.1 & 71.7 \\
\hline Deflexión del flap & $\delta_{f}$ & [deg] & $25^{\circ}$ & $0^{\circ}$ & $0^{\circ}$ & $0^{\circ}$ & $0^{\circ}$ & $0^{\circ}$ \\
\hline Factor de carga & $\mathrm{n}$ & & 3.8 & 4.1 & 3.8 & -0.94 & -2.11 & -1.5 \\
\hline \multicolumn{9}{|l|}{ RESULTADOS } \\
\hline Ángulo de ataque & $a$ & [deg] & $8.14^{\circ}$ & $7.48^{\circ}$ & $6.52^{\circ}$ & $-6.62^{\circ}$ & $-11.2^{\circ}$ & $-19.5^{\circ}$ \\
\hline Sustentación ala & L & {$[\mathrm{kg}]$} & 2580.5 & 1780 & 2571.2 & -311.5 & -842.2 & -970.2 \\
\hline Arrastre ala & D & [kg] & 247.7 & 100.17 & 136.5 & 20.7 & 31.9 & 59.9 \\
\hline Momento ala & $M_{a c}$ & [kg.m] & -419 & -205.2 & -320.6 & -320.6 & -205.2 & -99.2 \\
\hline Carga empenaje & $\mathbf{P}$ & {$[\mathrm{kg}]$} & 52.9 & -8.84 & 62.2 & -94.5 & -69.3 & -69.3 \\
\hline Fuerza normal al ala & $F_{z}$ & {$[\mathrm{~kg}]$} & 2589.5 & 1777.9 & 2570.1 & -311.9 & -832.3 & -934.5 \\
\hline Fuerza transversal al ala & $F_{x}$ & [kg] & -120 & -132.6 & -156.6 & -15.3 & -132.4 & -267.3 \\
\hline Fuerza normal al empenaje & $F_{z}$ & {$[\mathrm{~kg}]$} & 52.4 & -8.77 & 61.8 & -93.9 & -68 & -65.3 \\
\hline
\end{tabular}

Fuente: elaboración propia por medio de las herramientas Matlab ${ }^{\circledast}$ y Excel ${ }^{\circledast}$.

\section{Distribución de carga a lo largo de la envergadura}

Cargas simétricas

Puesto que se trata de un avión subsónico de bajas velocidades, una aproximación razonable es asumir que la combinación ala-fuselaje genera esencialmente la misma sustentación que el ala sola, en otras palabras, la sustentación es generada también por aquella parte del ala embebida en el fuselaje (Anderson, 1999) (ver figura 9).
La distribución de la sustentación es obtenida por medio de un programa escrito en Matlab ${ }^{\circledR}$, que implementa la conocida Teoría de la Línea de Sustentación (Lifting Line Theory), para este caso, el método convencional fue modificado para tener en cuenta la presencia del flap. Una vez obtenida la sustentación, esta se transforma en fuerza normal por medio de la ecuación (8). Los resultados para las dos condiciones más críticas son presentados en las figuras 10 y 11 :

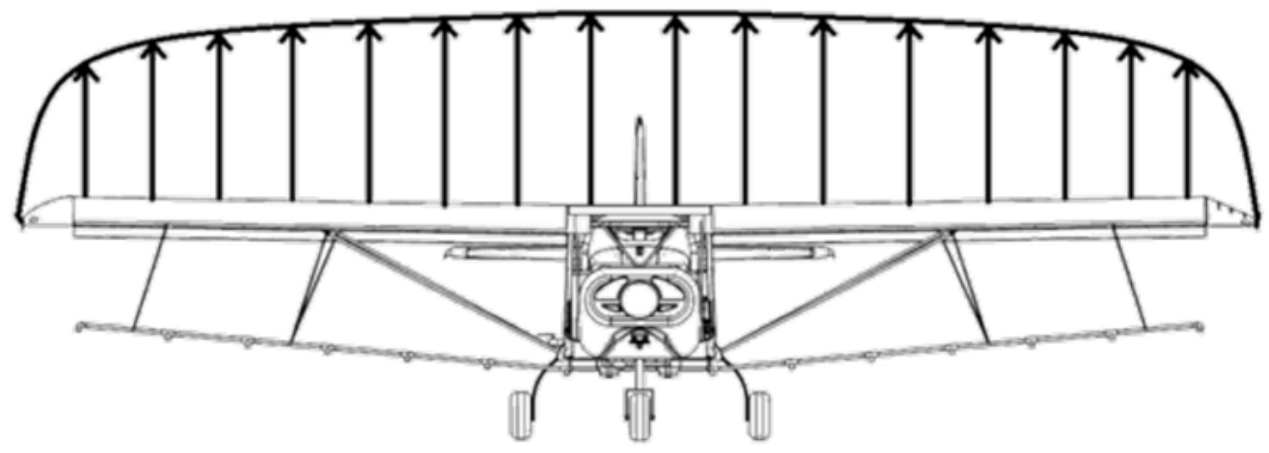

Figura 9. Distribución de la sustentación a lo largo del fuselaje Fuente: elaboración propia. 


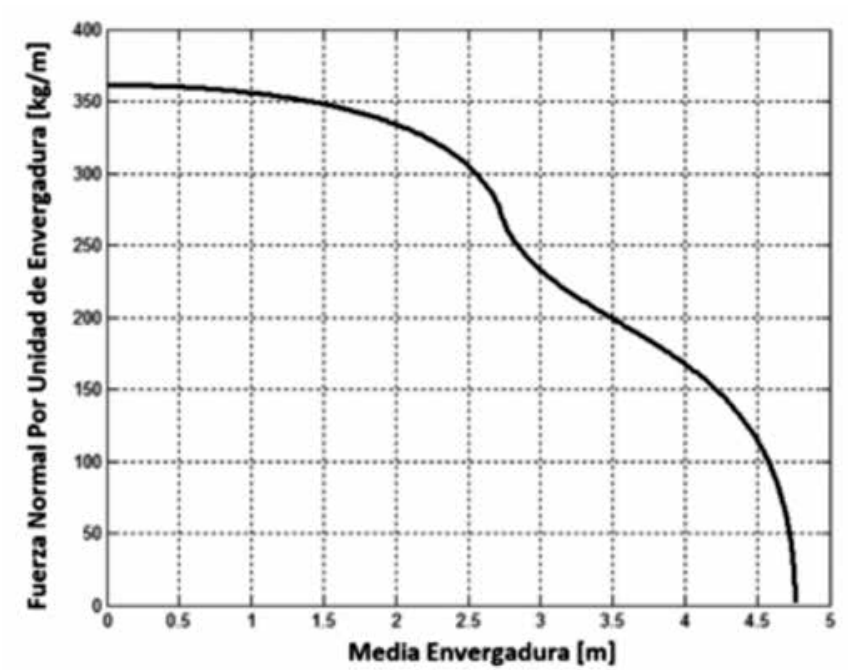

Figura 10. Distribución de fuerzas para la condición (A). ( $V=95.9$ [mph], $a=8.14^{\circ}, \delta_{f}=25^{\circ}$ )

Fuente: elaboración propia por medio de las herramientas Matlab ${ }^{\oplus}$ y Excel $^{\circledast}$.

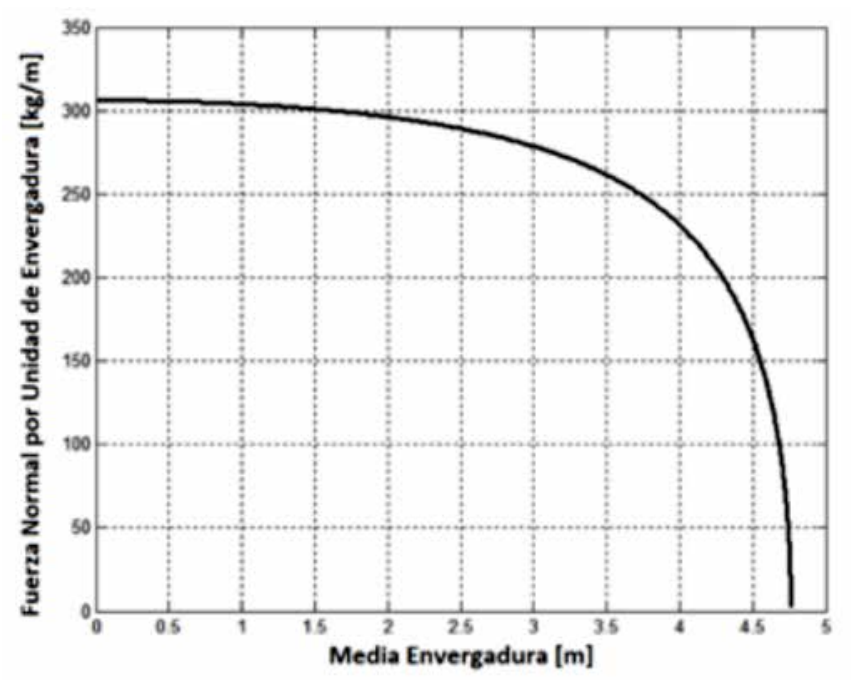

Figura 11. Distribución de fuerzas para la condición (D). ( $(V=128.8$ [mph], $a=6.52^{\circ},=0^{\circ}$ )

Fuente: elaboración propia por medio de las herramientas Matlab ${ }^{\circledast}$ y Excel $^{\circledast}$.
Condición especial para sustentación y factor de carga negativo (Rear Lift Truss)

La velocidad de cálculo a la cual se debe evaluar el flujo de aire reverso que causaría un factor de carga negativo está definido en CS-VLA 369 como:

$$
V=0.65 * \sqrt{\frac{M * g}{S}}+4.47
$$

Para un peso máximo de 693 kg.

$$
V=19.9[\mathrm{~m} / \mathrm{s}]
$$

La distribución de esta sustentación negativa se obtiene empleando el mismo código que para la distribución positiva, introduciendo como dato de entrada la velocidad calculada y probando diferentes ángulos de ataque hasta obtener el C negativo máximo de -0.8, de acuerdo con lo requerido en CS-VLA 369 (b).

Como se evidencia en la tabla 8, la condición más crítica para factores de carga negativo es el punto $(G)$ del diagrama $\vee$-n, donde el ala estaría sujeta a una fuerza normal hacia abajo de $934.5 \mathrm{~kg}$.

Según lo establecido en la Advisory Circular AC 23-19A (FAA, 2007) los struts que sostienen el ala generalmente trabajan a tensión, excepto durante maniobras con fuerzas $G$ negativas, como vuelos invertidos o ráfagas de viento descendentes, en estos casos, los struts trabajan a compresión y pueden estar sujetos al fenómeno de columna de Euler; razón por la cual, pese a que en ningún momento el WA500-AG le es permitido vuelo invertido, es importante considerar esta condición (ver figura 12).

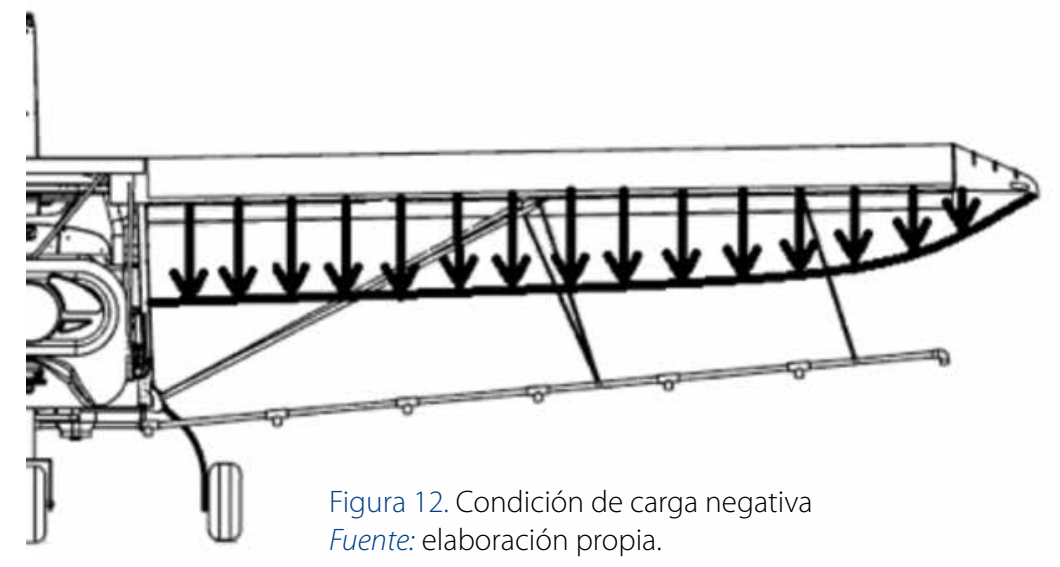


La distribución de carga negativa se grafica tanto para la condición (G) como para condición la especial de Rear Lift Truss, con el objetivo de compararlas e identificar el caso más crítico (figura 13):

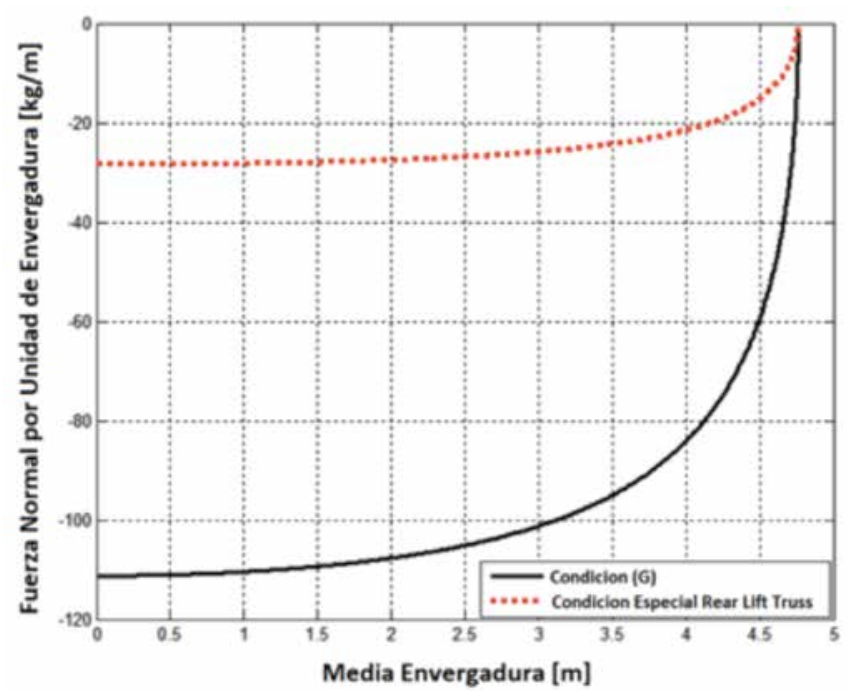

Figura 13. Distribución de cargas negativas a lo largo de la envergadura

Fuente: elaboración propia por medio de las herramientas Mat$1 a b^{\oplus}$ y Excel $^{\oplus}$.

Como se observa, la condición más crítica para carga negativa sería la condición (G) del diagrama V-n.

\section{Cargas asimétricas}

Los requerimientos de cargas asimétricas son evaluados de acuerdo con lo establecido en la norma: numerales CS-VLA 347, 349 y 455. El ala en sí, solo es afectada asimétricamente en los movimientos de alabeo (roll) que resultan de aplicar alerones.

Aquellas condiciones de alabeo que se deben considerar para el estudio, según lo requerido son:

Dos tercios del máximo factor de carga positivo en combinación con:

a. Deflexión máxima de alerón a velocidad $\mathrm{V}_{\mathrm{a}}$

b. Deflexión suficiente de alerón a Vc, tal que se alcance una tasa de alabeo igual a la obtenida en (a).

c. Deflexión suficiente de alerón a $\mathrm{Vd}$, tal que se alcance una tasa de alabeo no menor a 1/3 de la obtenida en (a).

Las condiciones de la aeronave volando a velocidad $V_{a}$ se encuentran en la tabla 8 , y la tasa de alabeo estable se determina por medio de la siguiente expresión (Gudmundsson, 2011):

$$
\frac{p * b}{2 * V}=-\frac{C_{l_{\delta a}}}{C_{l_{p}}} * \delta_{a}
$$

Siendo:

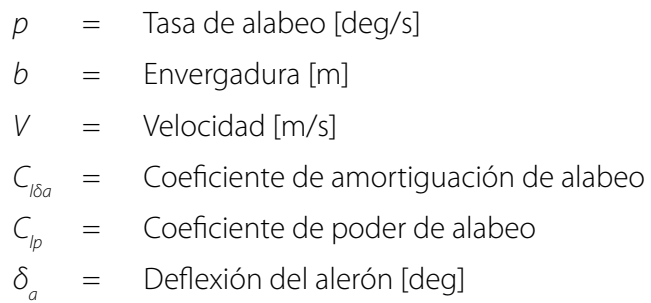

Los coeficientes de amortiguación y de poder de alabeo se calculan para un ala rectangular por medio de:

$$
C_{l_{\delta a}}=\frac{c_{l_{\delta a}} *\left(b_{2}^{2}-b_{1}^{2}\right)}{b^{2}}
$$

$$
C_{l_{p}}=-\frac{c_{l_{\alpha}}+c_{d_{o}}}{6}
$$

Donde los correspondientes términos de cada ecuación son (WACSA S.A.S, 2015):

$$
\begin{aligned}
& c_{1 \delta a}=\begin{array}{l}
\text { Coeficiente de incremento de sustentación debido a } \\
\text { la deflexión del alerón }=3.45\left[\mathrm{Rad}^{-1}\right] .
\end{array} \\
& c_{1 a}=\begin{array}{l}
\text { Pendiente curva de sustentación de la sección }= \\
6.646\left[\mathrm{Rad}^{-1}\right] .
\end{array} \\
& c_{d 0}=\begin{array}{l}
\text { Coeficiente de arrastre a cero sustentación }= \\
0.00725 .
\end{array} \\
& b_{1}^{2}=\begin{array}{l}
\text { Posición del borde interno del alerón desde la raíz } \\
\text { del ala }=2.74[\mathrm{~m}] .
\end{array} \\
& b_{2}^{2}=\begin{array}{l}
\text { Posición del borde externo del alerón desde la raíz } \\
\text { del ala }=4.38[\mathrm{~m}] .
\end{array}
\end{aligned}
$$

Con lo cual, volando a $V_{a}(42.88 \mathrm{~m} / \mathrm{s})$ y aplicando full alerón $\left(\delta=15^{\circ}\right)$, la tasa de alabeo de la aeronave sería aproximadamente:

$$
p=54.1\left[\frac{\operatorname{deg}}{s}\right]
$$

Manteniendo este valor para p, se resuelve la ecuación (11) para la deflexión de alerón necesaria en cada una de las condiciones faltantes (tabla 9): 
CIENCIA Y PODER AÉREO | ISSN 1909-7050 | E-ISSN 2389-9468 | Vol. 13 | Núm. 2 | Jul - Dic 2018 | Escuela de Postgrados de la Fuerza Aérea Colombiana | pp $28-47$

Tabla 9.

Condiciones de carga asimétrica

\begin{tabular}{lccc}
\hline CONDICIÓN & $\begin{array}{c}\text { Velocidad } \\
{[\mathrm{m} / \mathrm{s}]}\end{array}$ & $\begin{array}{c}\text { Deflexión de } \\
\text { alerón [deg] }\end{array}$ & $\begin{array}{c}\text { Tasa de } \\
\text { alabeo } \\
{[\mathbf{d e g} / \mathbf{s}]}\end{array}$ \\
\hline Full deflexión a $V_{a}$ & 42.88 & $15^{\circ}$ & 54.1 \\
\hline Alabeo a $V_{c}$ & 46.08 & $13.9^{\circ}$ & 54.1 \\
\hline Alabeo a $V_{d}$ & 57.6 & $11.2^{\circ}$ & 18 \\
\hline
\end{tabular}

Fuente: elaboración propia.

Las distribuciones de fuerza a lo largo de la envergadura para las condiciones asimétricas son graficadas y contrastadas con las condiciones más críticas del diagrama V-n (figura 10 y figura 11). Se observa que ninguna de las condiciones de carga asimétricas es más crítica que las de la envolvente de vuelo, luego, para propósitos de prueba estructural estática, la condición simulada para maniobra positiva es la (A) (figura 14):

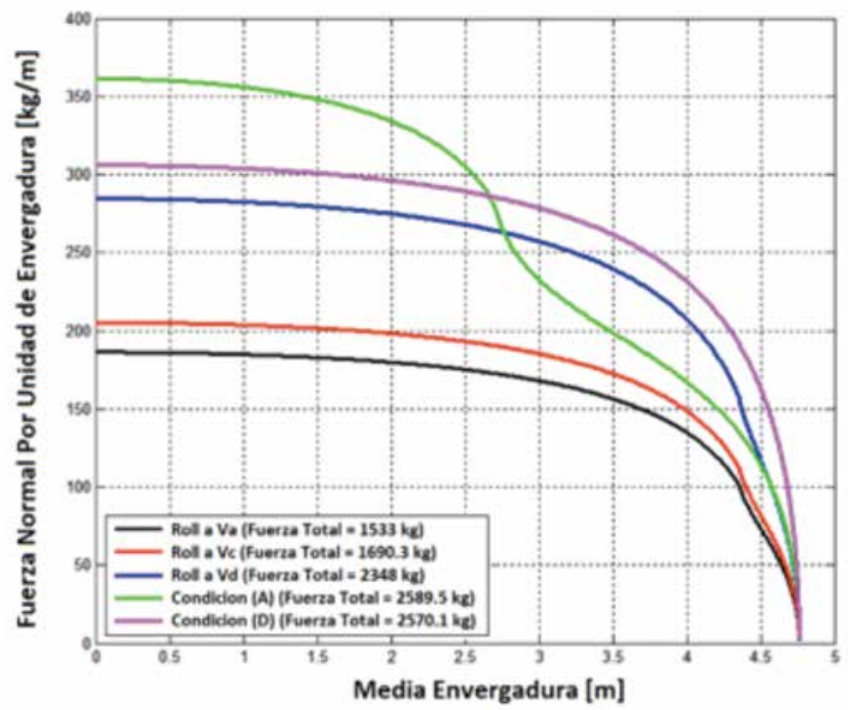

Figura 14. Comparación de las diferentes condiciones de alabeo con los puntos más críticos del diagrama V-n

Fuente: elaboración propia por medio de las herramientas Matlab ${ }^{\circledast}$ y Excel $^{\oplus}$

\section{Pruebas estructurales}

\section{Requerimientos}

Todos los anteriores valores corresponden a cargas límites, es decir, aquellas cargas máximas a las que estaría sometida la aeronave en condiciones normales de operación. En aeronáutica se define además la carga última, la cual es la límite multiplicada por un factor de seguridad, que para el caso de la norma CS-VLA está establecido en un valor igual a 1.5.

La normativa en su numeral CS-VLA 305 requiere que:

a. La estructura debe ser capaz de soportar las cargas límites sin presentar detrimento ni deformaciones permanentes.

b. La estructura deber ser capaz de soportar sin fallas las cargas últimas por al menos tres segundos.

El espécimen de prueba (ala) es sometido a las dos condiciones más críticas identificadas para cargas positiva y negativa de la envolvente de vuelo, que son, respectivamente: punto $(A)$ y punto $(G)$, como se aprecia en la tabla 10 .

Tabla 10

Valores de carga preliminares para prueba estructural

\begin{tabular}{ccccc}
\hline Condición & $\begin{array}{c}\text { Carga } \\
\text { total } \\
{[\mathbf{k g}]}\end{array}$ & $\begin{array}{c}\text { Carga por } \\
\text { ala (límite) } \\
{[\mathbf{k g}]}\end{array}$ & $\begin{array}{c}\text { Factor de } \\
\text { seguridad }\end{array}$ & $\begin{array}{c}\text { Carga } \\
\text { última } \\
{[\mathbf{k g}]}\end{array}$ \\
\hline (A) & 2589.5 & 1294.7 & 1.5 & 1942 \\
\hline (G) & -934.5 & 467.2 & 1.5 & 700.1 \\
\hline
\end{tabular}

Fuente: elaboración propia.

\section{Cargas no consideradas}

Por la forma constructiva del montaje, existe una fracción de la fuerza que no se toma en cuenta al momento de cargar la estructura.

Por un lado, la porción de la sustentación generada por el fuselaje, que para la condición (A) equivale aproximadamente a 202 kg y 62 kg para la condición (G) (figura 15).

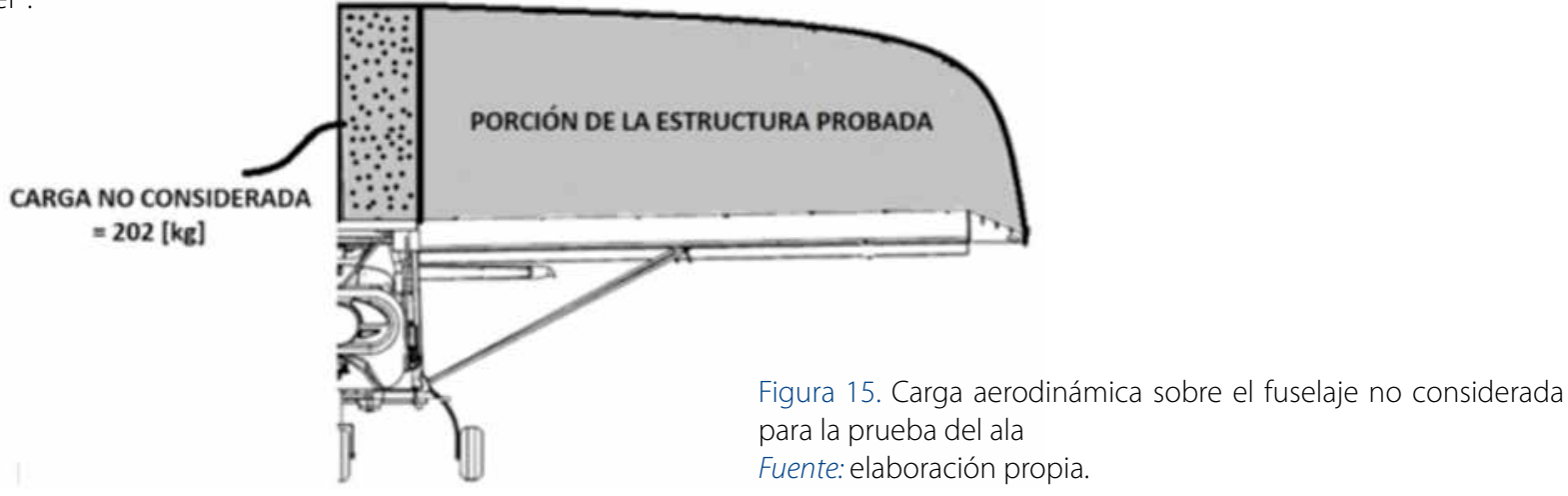


Por otra parte, la estructura del ala pesa aproximadamente $20 \mathrm{~kg}$; ya que la misma se prueba en posición invertida (figura 18 a), este valor debe sustraerse a la carga total, pues el peso es una fuerza contraria a la sustentación. La misma consideración se tiene con el peso de las placas de madera utilizadas como apoyo para las bolsas de arena $(31 \mathrm{~kg})$. Con todo lo anterior, la carga total empleada en la prueba es la que se presenta en la tabla 11.

Tabla 11.

Valores de carga finales para prueba estructural

\begin{tabular}{ccc}
\hline Condición & Carga límite $[\mathrm{kg}]$ & Carga última $[\mathrm{kg}]$ \\
\hline (A) & 1042 & 1563 \\
\hline (G) & 405 & 607.5 \\
\hline
\end{tabular}

Fuente: elaboración propia.

Distribución de pesos a lo largo de la envergadura Las curvas de distribución de sustentación de la figura 10 y 13 son aproximadas a secciones rectangulares, que en el espécimen de prueba representa siete secciones en las cuales está dividida el ala, como se muestra en el figura 16 y 17:

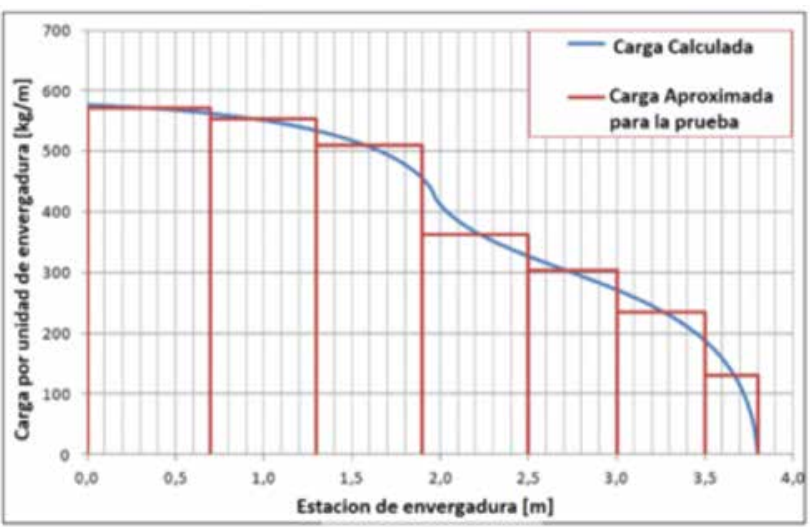

Figura 16. Distribución de carga última para maniobra positiva Fuente: elaboración propia por medio de las herramientas Mat$\mathrm{lab}^{\oplus}$ y Excel $^{\oplus}$

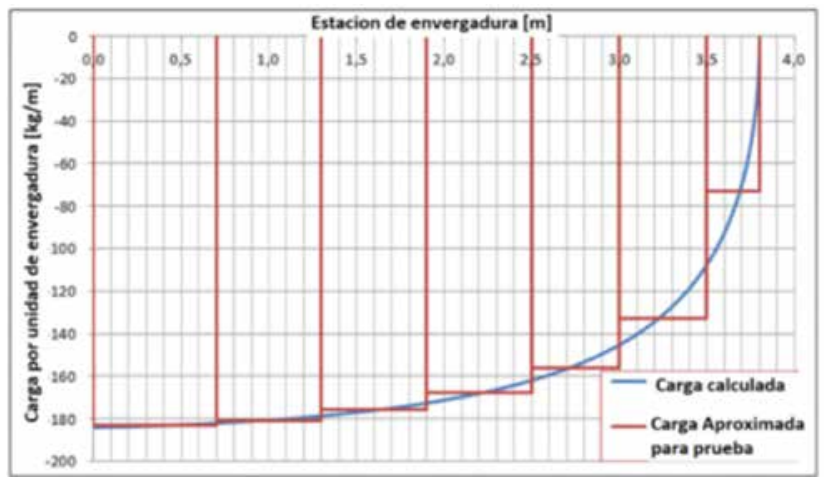

Figura 17. Distribución de carga ultima para maniobra negativa Fuente: elaboración propia por medio de las herramientas Matlab $^{\oplus}$ y Excel $^{\oplus}$.

\section{Montaje}

Para evaluar las dos condiciones más críticas, se diseña un banco de pruebas sobre el cual es ensamblada el ala, la cual, a su vez, es una copia exacta de la estructura instalada en la aeronave final (figura 18).

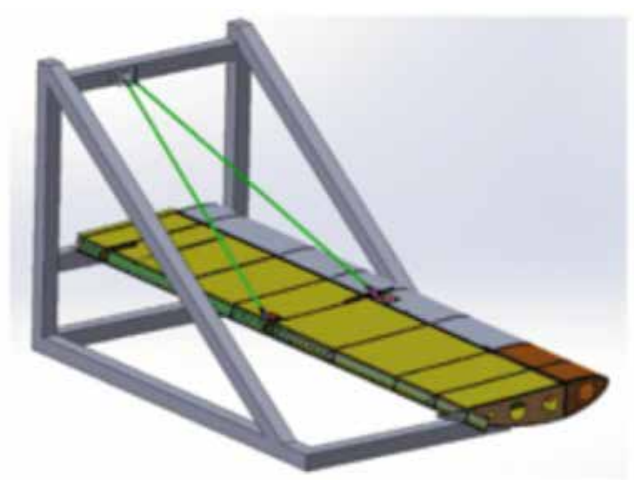

(a)

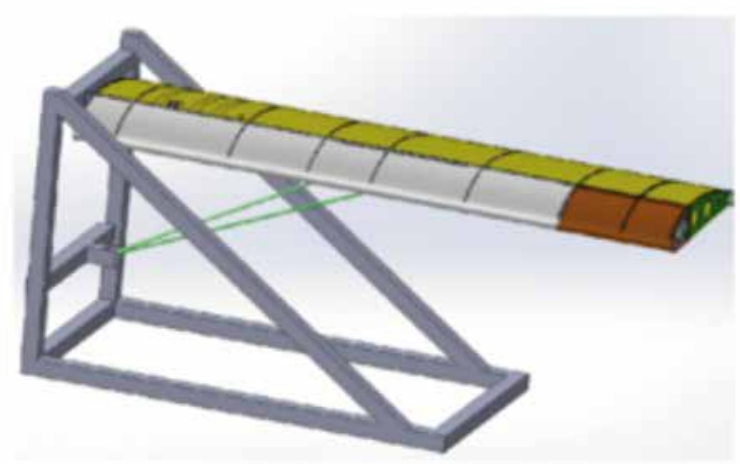

(b)

Figura 18. Ilustración del montaje de pruebas. (a) Para carga positiva, (b) para carga negativa

Fuente: elaboración propia.

\section{Descripción del procedimiento}

La carga es simulada por medio de bolsas de arena de 10, 12,20 y 24 kg, identificadas por colores amarillo, rojo, azul y verde, respectivamente. La semiala es cubierta con paneles de madera sobre los cuales se apoyan las bolsas, esto con el fin de evitar arrugar la piel y falsear los resultados de las vigas. Se utilizan varios paneles en lugar de un solo bloque para permitir que la estructura se flexione como lo haría en vuelo. Mientras se posicionan las bolsas de arena, el ala es sostenida por medio de un gato mecánico.

Inicialmente, el ala es cargada y descargada a la mitad de la carga límite, con el fin de permitir el asentamiento de la estructura, que al ser nueva puede presentar pequeñas holguras que confundirían las mediciones de desplazamiento.

Después del asentamiento, se define la referencia cero a partir de la cual se toman las mediciones (figura 19). 
Se somete el ala a carga límite, se descarga y se registra cualquier deformación permanente.

Finalmente, se somete la estructura a la carga última por los tres segundos requeridos por norma, y se verifica que no colapse en este lapso de tiempo.

Estos procedimientos se llevan a cabo para ambas condiciones simuladas, esto es, maniobra negativa y positiva (figuras 20 y 21).

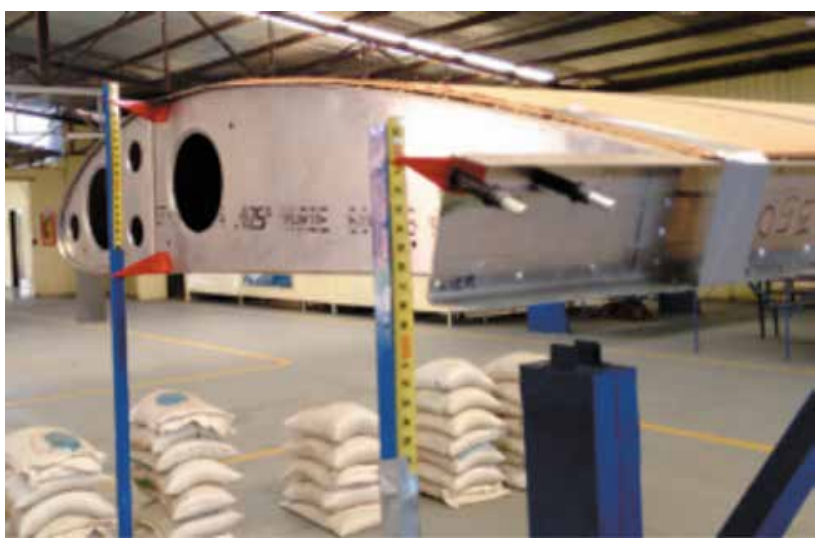

Figura 19. Método real de medición de las deflexiones Fuente: elaboración propia.

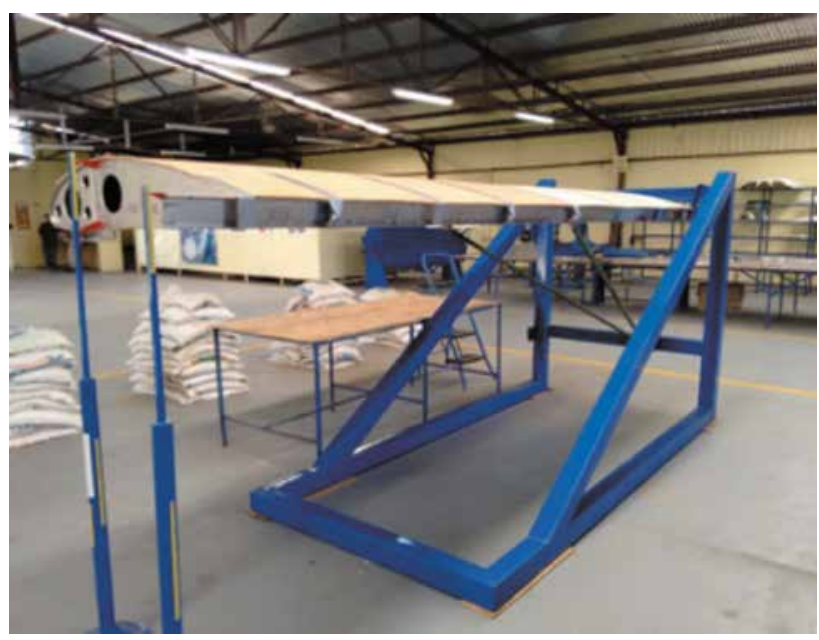

Figura 20. Montaje para carga de maniobra negativa Fuente: elaboración propia.

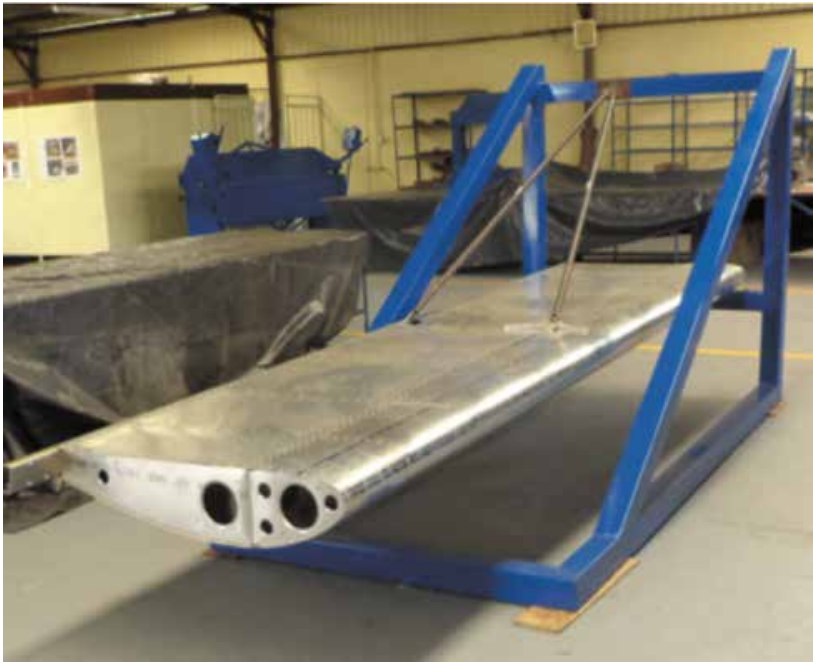

Figura 21. Montaje para carga de maniobra positiva Fuente: elaboración propia.

\section{Resultados}

Maniobra negativa. Carga límite

Después de aplicar la carga de asentamiento y tomar la referencia cero, la estructura es cargada hasta la condición límite, el gato mecánico bajado y la deflexión registrada. La deformación (imperceptible a la vista) fue de solo $2 \mathrm{~mm}$. Valor despreciable que no representa una amenaza para la integridad estructural (figura 22).

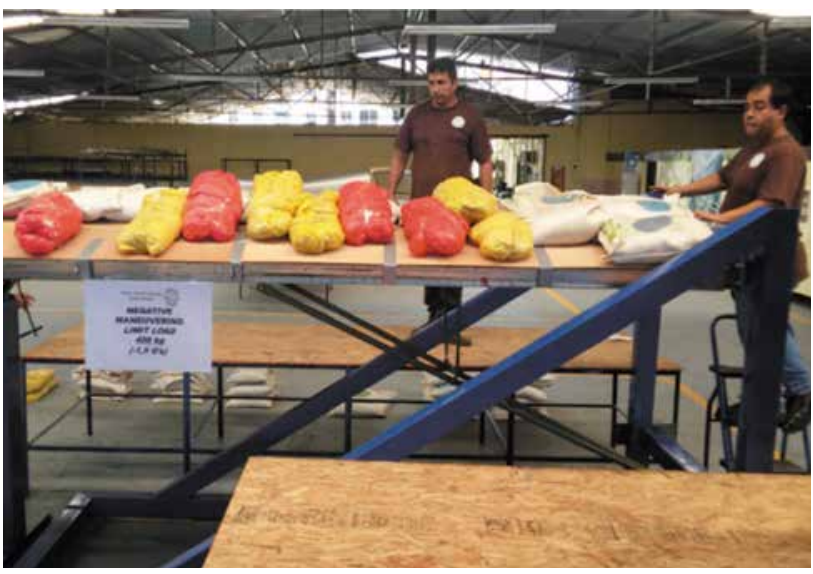

Figura 22. Carga límite negativa (406 kg =-1.5 G) Fuente: elaboración propia. 


\section{Carga última}

La carga última es aplicada, y a medida que el gato mecánico es bajado se nota un fenómeno de pandeo (buckling) en los struts, lo cual obliga a detener la prueba (figura 23).

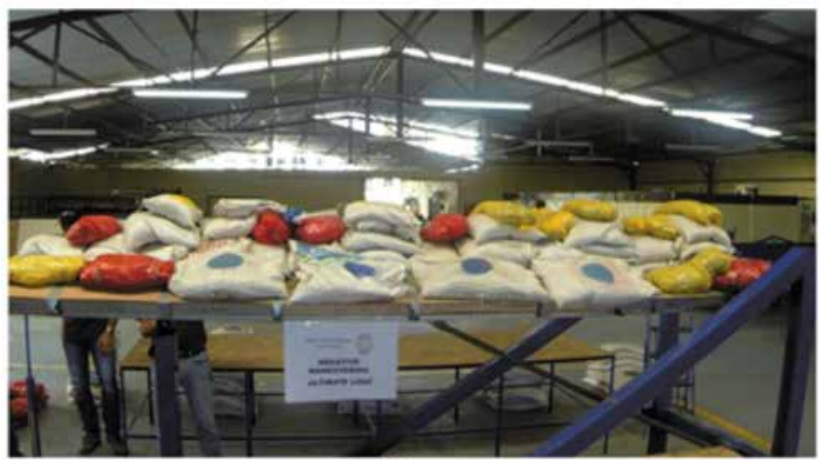

(a)

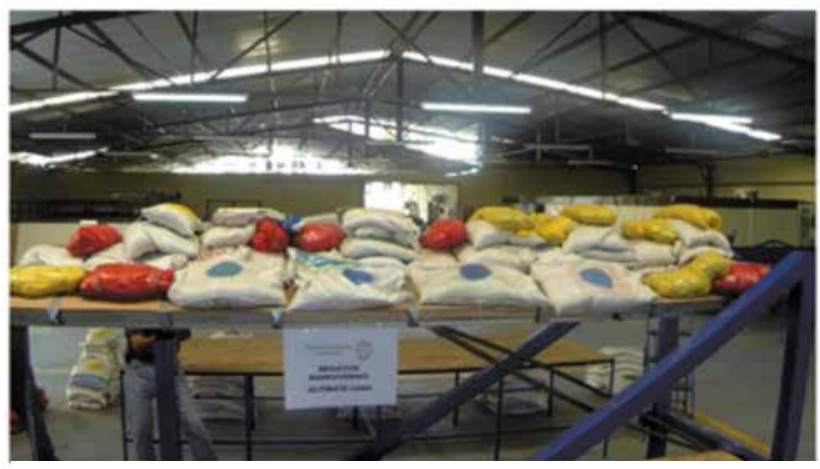

(b)

Figura 23. (a) Comienzo de la prueba a carga última. (b) Pandeo de los struts

Fuente: elaboración propia.

La prueba a carga última se considera fallida, y se toma como acción correctiva la instalación de elementos denominados Jury Struts, con el objetivo de brindar más rigidez a los struts (figura 24).
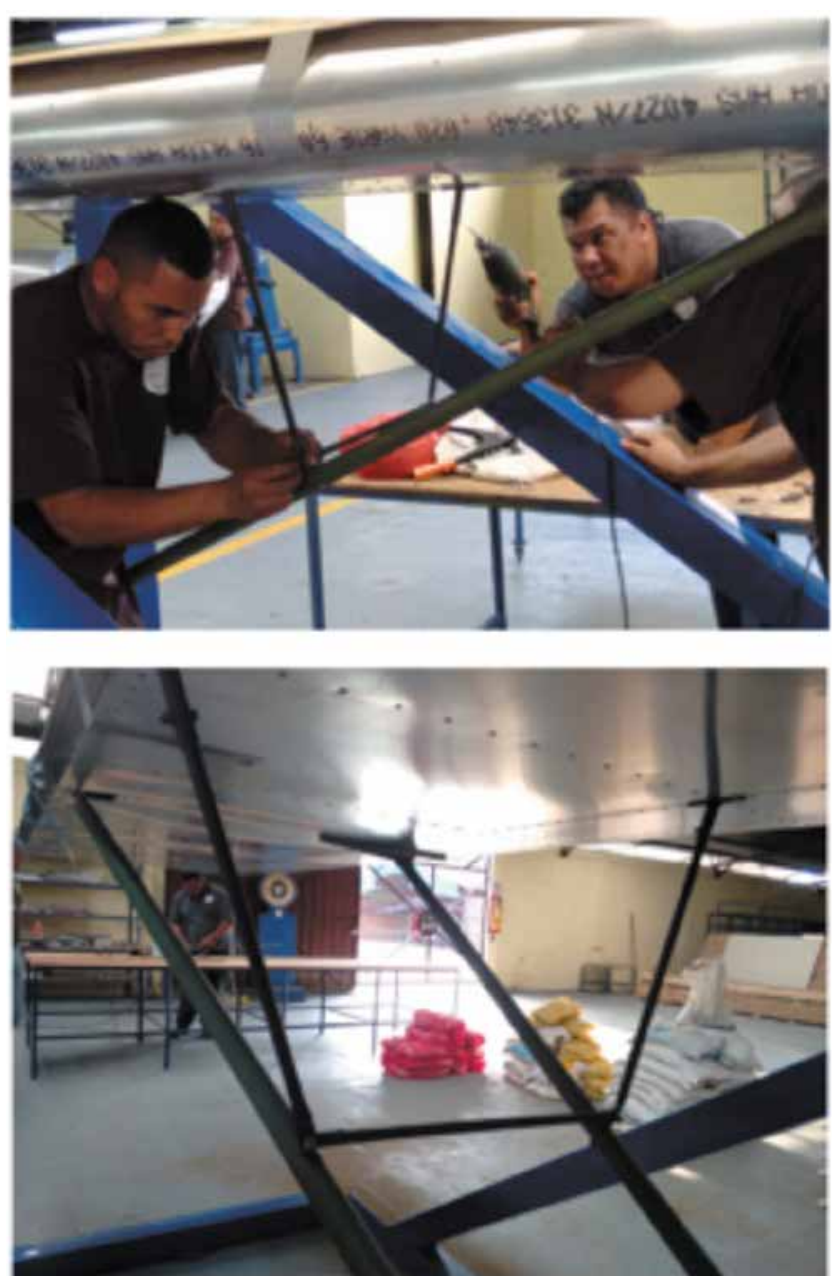

Figura 24. Instalación de Jury Struts Fuente: elaboración propia.

La prueba a carga última es repetida y la estructura soporta sin problemas por más de tres segundos, de acuerdo con lo requerido (figura 25).

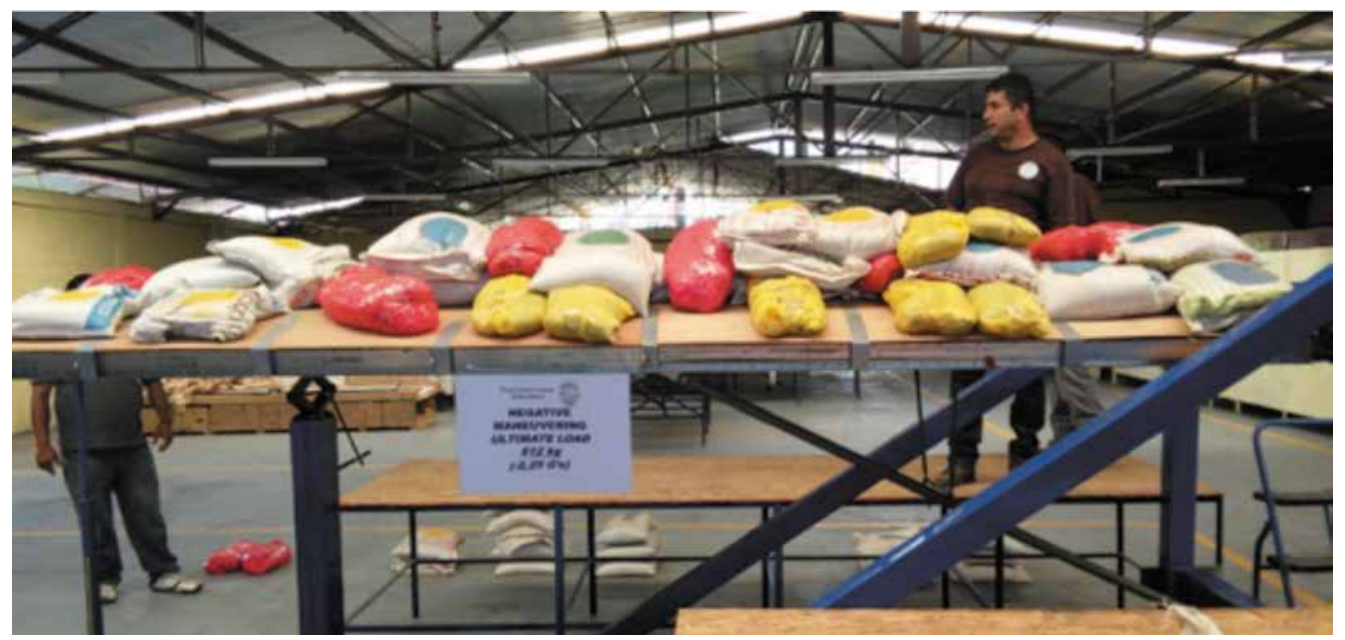

Figura 25. Carga última negativa $(612 \mathrm{~kg}=-2.25 \mathrm{G})$

Fuente: elaboración propia. 


\section{Maniobra positiva}

En una primera sesión, el ala demuestra soportar la carga límite presentando una deformación de solo $3 \mathrm{~mm}$. Al momento de aplicar la carga última y antes de contabilizar tres segundos, el punto de conexión del strut al ala colapsa, considerándose entonces una prueba fallida (figuras 26 y 27 ).

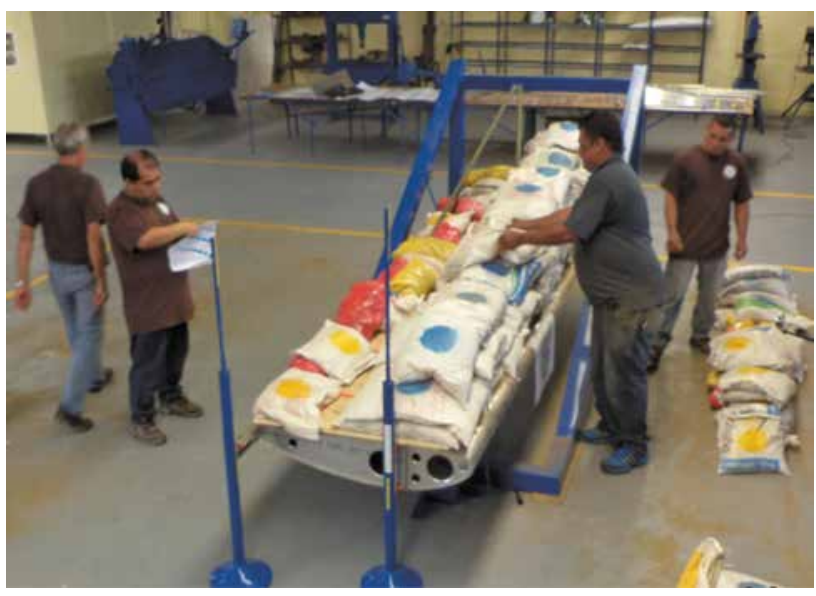

Figura 26. Aplicación de carga última positiva Fuente: elaboración propia.
Después de analizar el elemento colapsado y sopesado las posibles causas de fallo, la pieza se rediseña alineando los agujeros y aumentando las distancias a los bordes, como se ilustra en la figura 28.

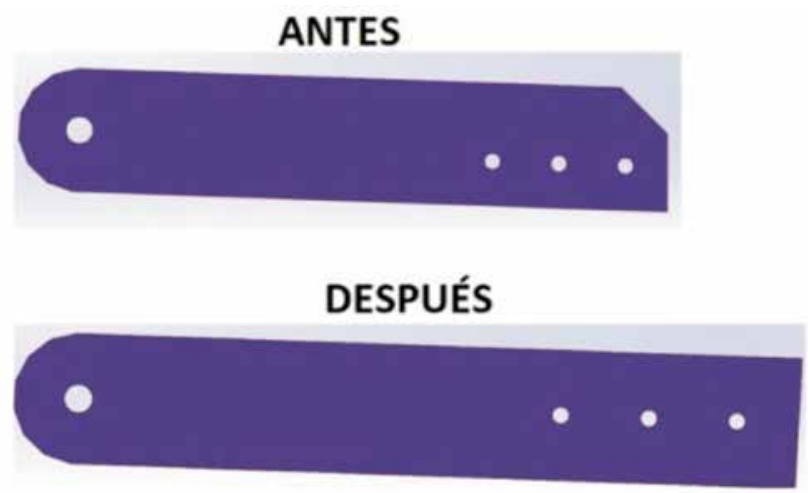

Figura 28. Modificación de la pieza fallida Fuente: elaboración propia.

Igualmente, otras zonas del ala son modificadas mediante refuerzos en $L$, y aumentando el número de costillas en la zona del tanque de combustible (figuras 29, 30 y 31).

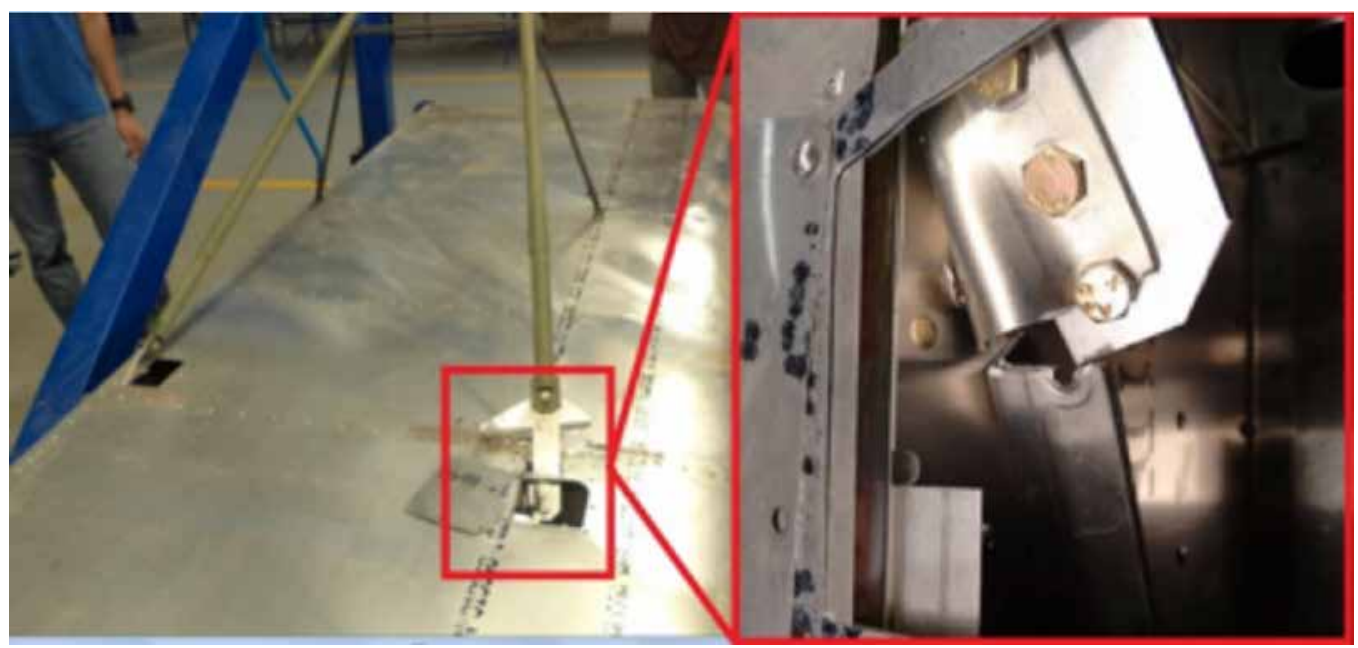

Figura 27. Elemento fallido bajo carga última: conexión entre el strut y el ala Fuente: elaboración propia. 
ANTES

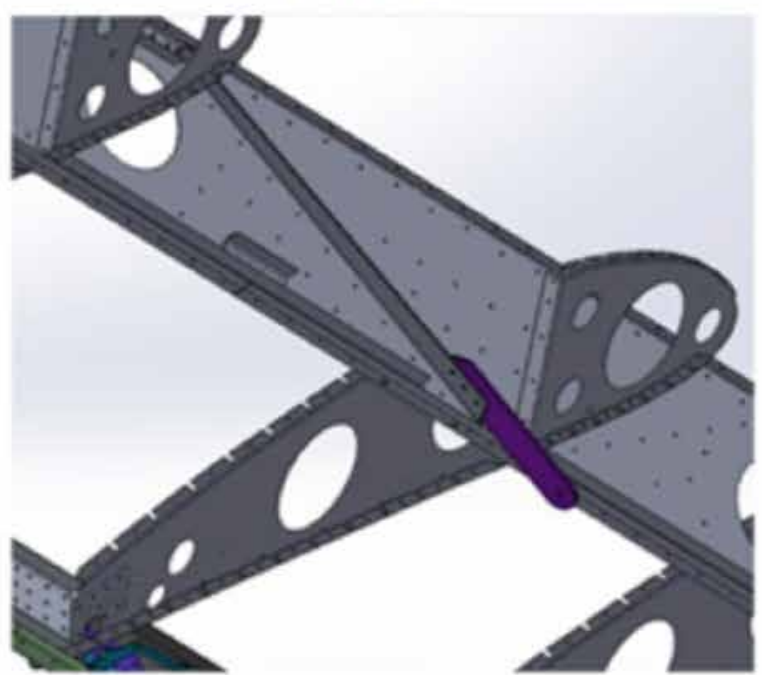

Figura 29. Refuerzo en la zona central del ala
Fuente: elaboración propia.

\section{ANTES}

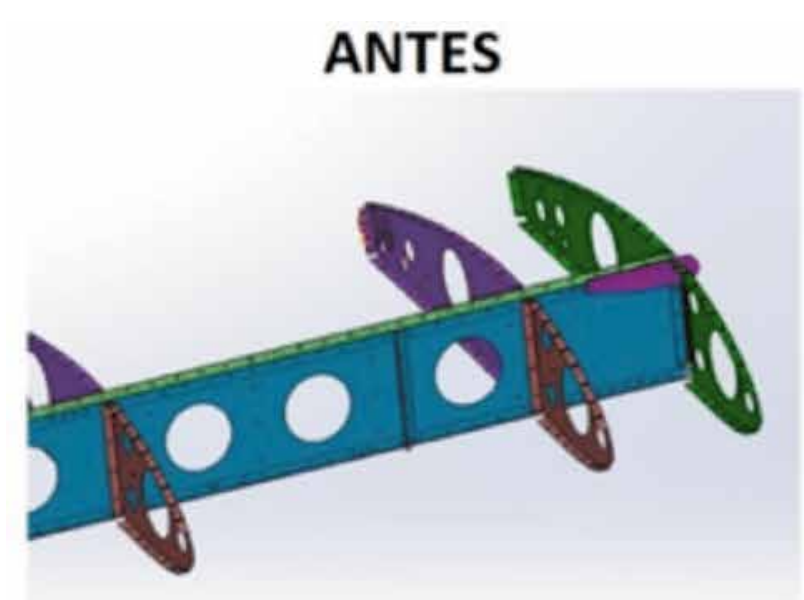

\section{DESPUÉS}

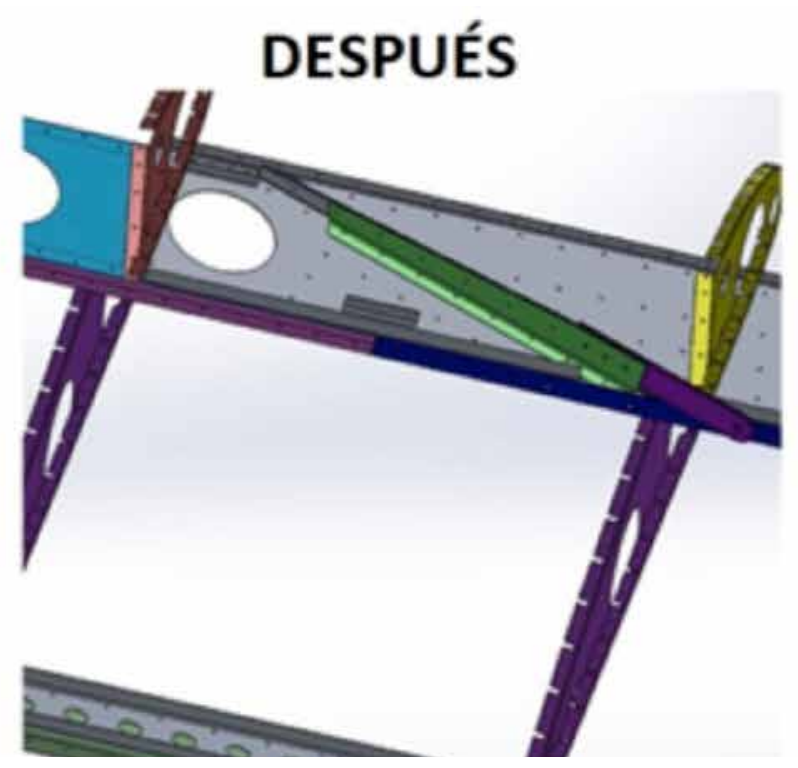

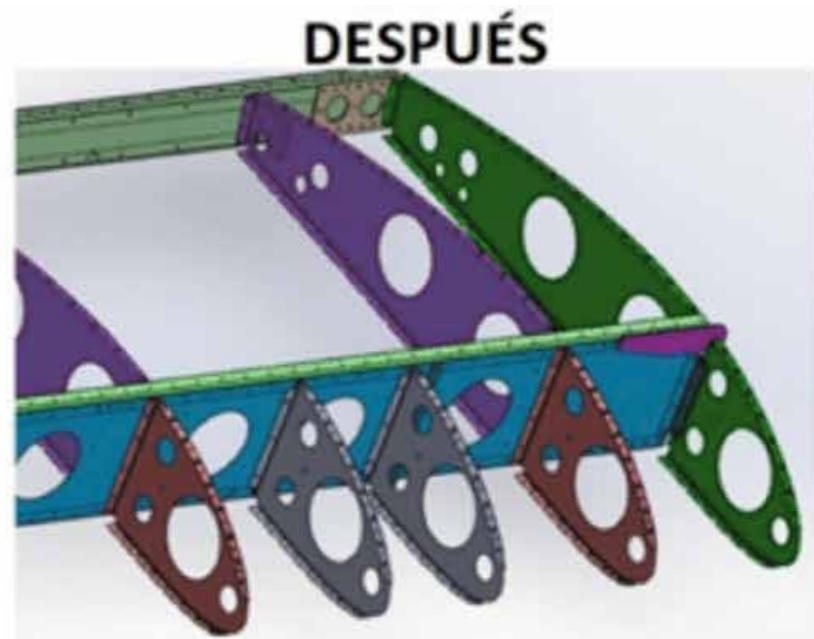

Figura 30. Aumento del número de costillas en la zona del tanque de combustible Fuente: elaboración propia.

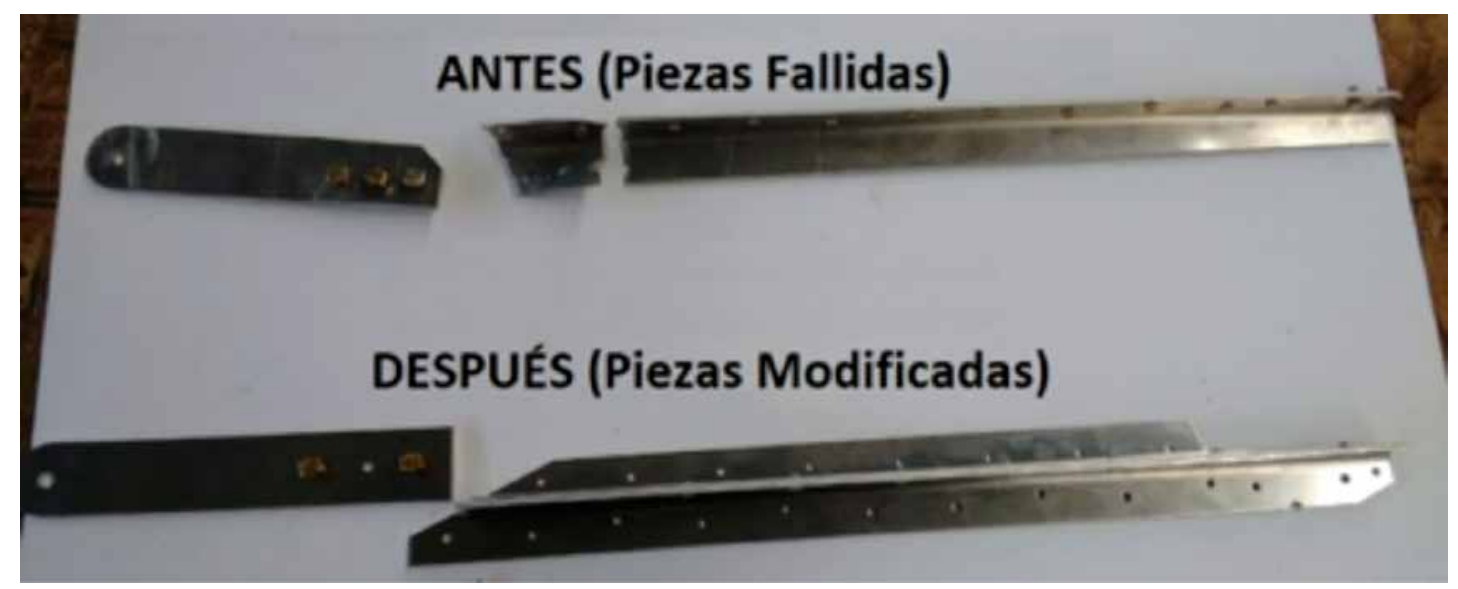

Figura 31. Fotografía de las piezas modificadas Fuente: elaboración propia. 


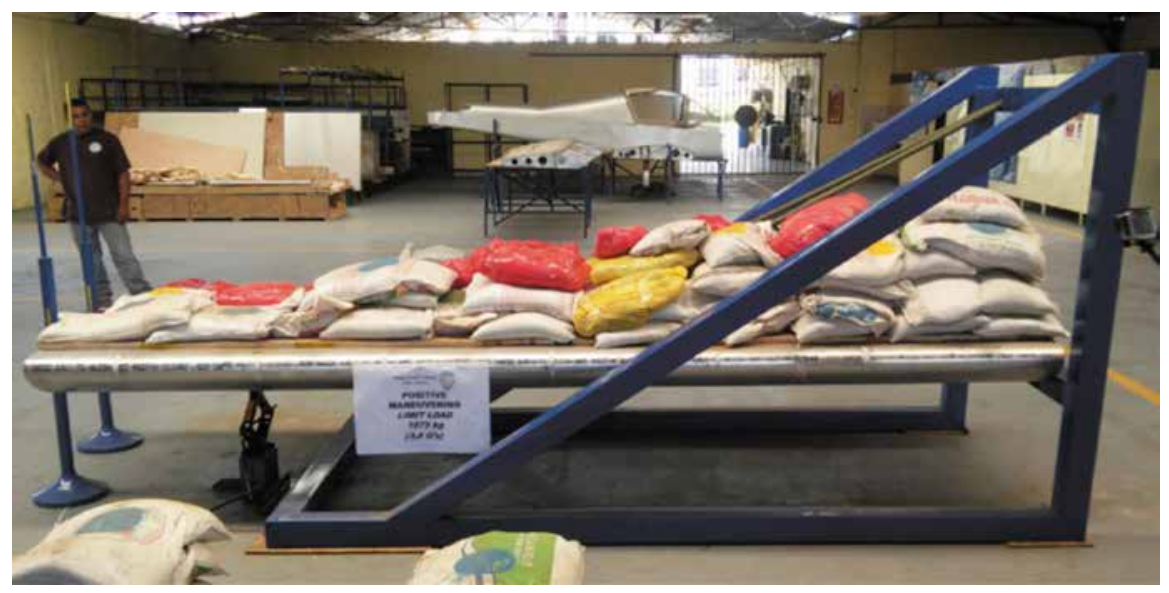

Figura 32. Carga límite positiva $(1075 \mathrm{~kg}=3.8 \mathrm{G})$

Fuente: elaboración propia.

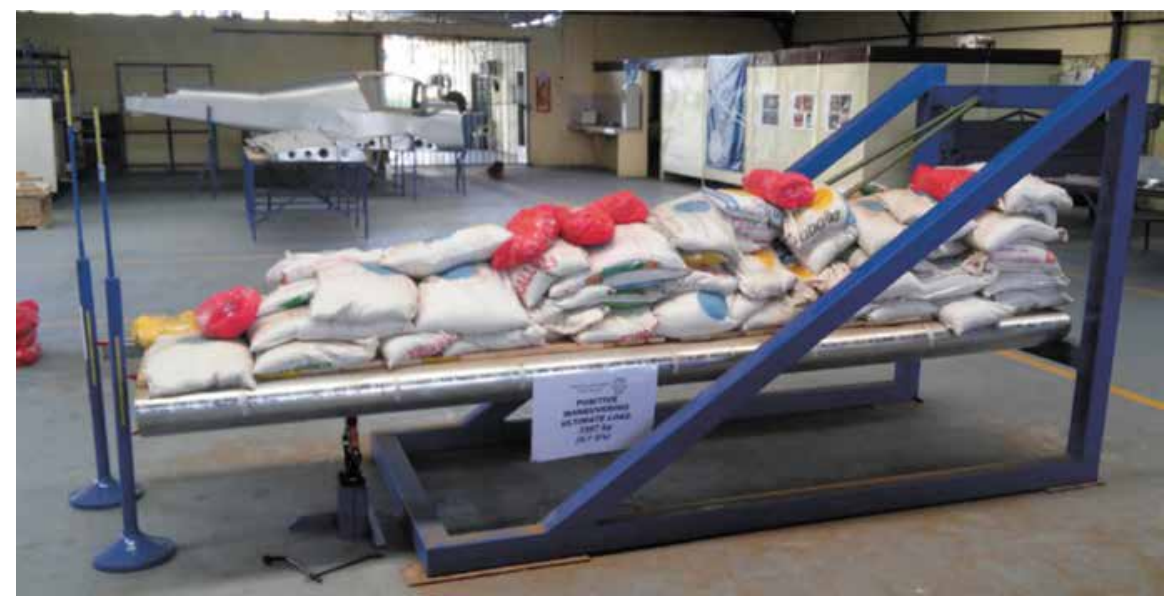

Figura 33. Carga última positiva $(1597 \mathrm{~kg}=5.7 \mathrm{G})$

Fuente: elaboración propia.

Una vez hechas las mejoras, en una segunda sesión se somete nuevamente la estructura a carga límite presentando una deformación de solo 4 mm (Figura 32).

Finalmente, la estructura también demuestra soportar la carga última por varios segundos sin colapsar, de acuerdo con lo requerido en la norma (figura 33).

\section{Conclusiones}

En este reporte de caso se ilustra parte del trabajo de certificación de la aeronave colombiana WA500-AG, particularmente del ala. Todo el proceso de análisis de cargas y pruebas estructurales conllevaron a resultados satisfactorios, donde la estructura presentó deformaciones despreciables y en los casos de carga última donde hubo falla, se identificaron las causas, se implementaron mejorías, se repitieron las pruebas y se mostró conformidad con la normativa. El rediseño de las piezas e instalación de Jury Struts son permitidas desde un punto de vista legal, ya que el diseño de la aeronave es propio, es decir, no son requeridas asesorías ni autorizaciones de fabricantes externos. Con esto, el presente artículo pretende demostrar las capacidades aeronáuticas de nuestro país, que no se limitan solamente a la fabricación de piezas o al ensamble y modificación de aeronaves extranjeras, sino que estamos capacitados para llevar a cabo todo un proceso de diseño, fabricación, y -recientemente- certificación de productos aeronáuticos nacionales.

Este artículo solo muestra parte del proceso de certificación, pero es importante destacar que estudios y pruebas semejantes se han elaborado para el estabilizador horizontal, vertical, bancada de motor, tren de aterrizaje, puntos de anclaje de sillas y cinturones de seguridad, superficies de control y tanques de combustible. Todos y cada uno auditados y en presencia de personal de la Aeronáutica Civil Colombiana (figura 35). 


\section{Referencias}

Aerocivil. (2012). Reglamentos Aeronauticos Colombianos - RAC 26. Colombia: Unidad Administrativa Especial de Aeronautica Civil.

Anderson, J. (1999). Aircraft Performance and Design. U.S.A: McGraw-Hill.

EASA. (2009). Certification Specifications For Very Light Aeroplanes, CS-VLA Book 1. European Aviation Safety Agency.

FAA. (2007). AC n. : 23-19A - Airframe Guide For Certification of Part 23 Airplanes. U.S.A: U.S. Federal Aviation Administration.
Gudmundsson, S. (2011). Aircraft Preliminary Handbook. Dayton Beach, Florida: Embry-Riddle Aeronautical University.

Megson, T. (2007). Aircrafts Structures for Engineering Students, (4 ed.). Jordan Hill, Oxford: Elsevier Aerospace Engineering Series.

OACl. (2010). Anexo 8 al convenio sobre aviación civililnternacional: aeronavegabilidad. Montreal, Canada: Organización de Aviación Civil Internacional.

WACSA S. A. S. (2015). WA500-AG Aerodynamic Data Determination. Unpublished. Cali, Colombia: World Aircraft Company South America S. A. S.

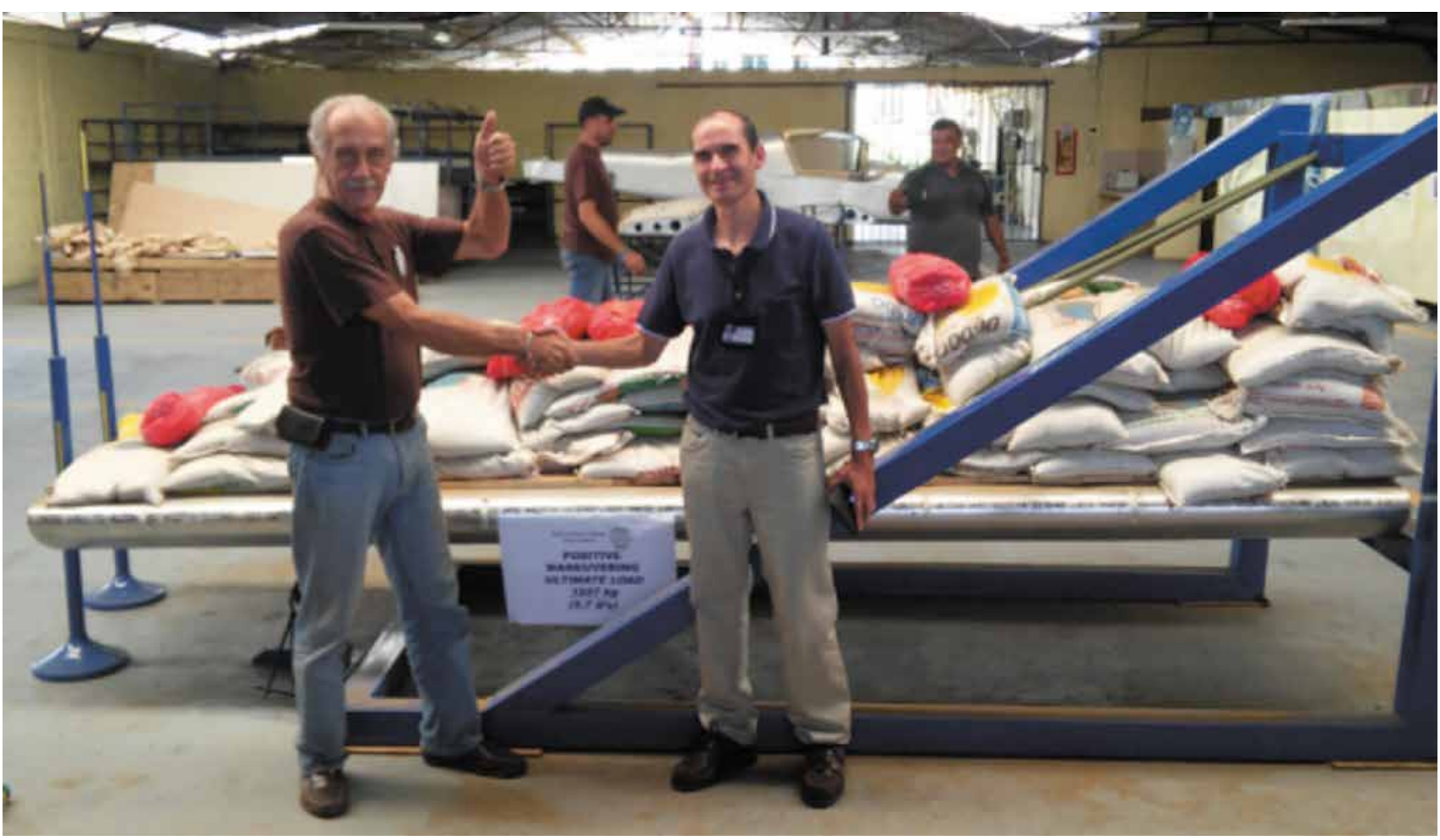

Figura 35. De izquierda a derecha: Máximo Tedesco (representante legal de WACSA S. A. S) y Jairo Sora (coordinador del grupo de certificación de productos aeronáuticos: Aeronáutica Civil Colombia)

Fuente: elaboración propia. 